\title{
Threshold Dynamics of an SIR Model with Nonlinear Incidence Rate and Age-Dependent Susceptibility
}

\author{
Junyuan Yang $\mathbb{D}^{1,2}$ and Xiaoyan Wang $\mathbb{D}^{3}$ \\ ${ }^{1}$ Complex Systems Research Center, Shanxi University, Taiyuan, Shanxi 030006, China \\ ${ }^{2}$ Shanxi Key Laboratory of Mathematical Techniques and Big Data Analysis on Disease Control and Prevention, Shanxi University, \\ Taiyuan, Shanxi 030006, China \\ ${ }^{3}$ School of Information Management, Shanxi University of Finance and Economics, Taiyuan, Shanxi 030006, China
}

Correspondence should be addressed to Junyuan Yang; yangjunyuan00@126.com

Received 14 May 2018; Accepted 17 July 2018; Published 14 October 2018

Academic Editor: Diego R. Amancio

Copyright (c) 2018 Junyuan Yang and Xiaoyan Wang. This is an open access article distributed under the Creative Commons Attribution License, which permits unrestricted use, distribution, and reproduction in any medium, provided the original work is properly cited.

\begin{abstract}
We propose an SIR epidemic model with different susceptibilities and nonlinear incidence rate. First, we obtain the existence and uniqueness of the system and the regularity of the solution semiflow based on some assumptions for the parameters. Then, we calculate the basic reproduction number, which is the spectral radius of the next-generation operator. Second, we investigate the existence and local stability of the steady states. Finally, we construct suitable Lyapunov functionals to strictly prove the global stability of the system, which are determined by the basic reproduction number $\mathscr{R}_{0}$ and some assumptions for the incidence rate.
\end{abstract}

\section{Introduction}

Mathematical modelling has been paid attention on investigating the intrinsic mechanisms of the disease prevalence. Many factors have been illustrated to affect dynamics of diseases spread. One of them is the susceptibility, which describes the fragility of individuals attacked during their lifetime. The susceptible ability is determined by individuals' behaviors and the immunity of individuals. As Brucellosis, humans associated with animals are more easy to be transmitted compared with ones apart from these kinds of jobs. There are few literatures $[1,2]$ studying this factor to affect the dynamics of systems. In this paper, we introduce susceptible age to evaluate the susceptibility for the modelling process.

Besides, incidence rates play an important role in the disease spread pattern. Compartment modelling method was firstly proposed by Kermack and McKendrick [3, 4]. The baseline models are SIS-type and SIR-type, respectively, in which $S$ denotes the susceptible individuals, $I$ represents the infectious individuals, and $R$ denotes the recovered individuals ( $N$ is the total population). From mathematical points of view, there are two classical forms: one is bilinear denoted and the other is standard incidence denoted by $\beta S I$ and $\beta S I / N$, where $\beta$ represents the transmission rate. As literatures indicated, the two incidence rates have distinct limitations, that is, the bilinear one is suitable for an initial phase of some disease spread, while the other one is applicable for the final phase. In order to overcome these defaults, Capasso and Serio in [5] introduced a saturated incidence rate $\beta S I / 1+\alpha I$ to connect the above two cases. There is no doubt that it is more realistic and is more meaningful than the old ones. Thereafter, a general case given by $f(S(t)) G(I(t))$ was introduced into the models (see, e.g., $[6,7])$. Furthermore, a general incidence rate written as $f(S, I)$ in $[8]$ had been used to investigate the global dynamics of SIR and SIRS epidemic models.

To our best knowledge, Magal et al. are the pioneers to investigate the global stability of the steady states for agestructured model by constructing Lyapunov functional in [9]. Since then, many researchers [10-13] have extensively studied the global behavior for age-structured epidemic models and bilinear incidence rates and the references therein. Since the complex structure for age-structured 
models, there are few references to establish global dynamics of epidemic models combining age structure and nonlinear incidence [14-17]. This issue has been becoming a challenging task for lacking well-established tools.

As we know, the recovered individuals can gain permanent disease-induced immunity for some diseases such as chickenpox, mumps, and measles. Motivated by what has been discussed, we integrate the susceptible age with a general incidence rate into an SIR model given as follows:

$$
\begin{aligned}
\frac{\partial s(t, a)}{\partial t}+\frac{\partial s(t, a)}{\partial a} & =-\mu s(t, a)-\beta(a) f(s(t, a), I(t)), \\
s(t, 0)= & \Lambda, \\
\frac{d I(t)}{d t}= & \int_{0}^{\infty} \beta(a) f(s(t, a), I(t)) d a \\
& -\left(\mu+\gamma+\delta_{1}\right) I(t), \\
\frac{d R(t)}{d t}= & \gamma I(t)-\mu R(t),
\end{aligned}
$$

with initial condition

$$
s(0, a)=s_{0}(a) \in L_{+}^{1}, I(0)=I_{0} \geq 0, R(0)=R_{0} \geq 0,
$$

where $L_{+}^{1}$ is the set of integrable functions from $(0, \infty)$ into $\mathbb{R}_{+}=[0, \infty)$. The total population is classified into three classes: susceptible $s(t, a)$, infected $I(t)$, and recovered $R(t)$. $\mu$ denotes the natural death rate, $\delta_{1}$ denotes the death rate due to disease, and $\gamma$ is the recovery rate. $\beta(a)$ is the transmission rate with respect to susceptible age $a$; $f(s(t, a), I(t))$ represents the transmission incidence and it concludes many common forms such as $\beta(a) s(t, a) I(t), \beta(a) s(t, a) I(t) /(1+\alpha$ $I)$, and $\beta(a) s(t, a) I(t) /\left(1+\alpha_{1} s(t, a)+\alpha_{2} I(t)+\alpha_{3}(a) s(t, a) I\right.$ $(t))$. All the parameters satisfy the following assumptions:

Assumption 1.1. (A1) $\Lambda$ and $\mu$ are positive; $\delta_{1}$ and $\gamma$ are nonnegative.

(A2) The function $\beta(a) \in C_{B U}\left(\mathbb{R}_{+}, \mathbb{R}_{+}\right) \cap L^{\infty}\left(\mathbb{R}_{+}\right)$where $C_{B U}\left(\mathbb{R}_{+}, \mathbb{R}_{+}\right)$is the set of all bounded and uniformly continuous functions from $\mathbb{R}_{+}$to $\mathbb{R}_{+}$. Denote

$$
\bar{\beta}=\operatorname{ess} \sup _{a \in \mathbb{R}_{+}} \beta(a) .
$$

(A3) For $s, I \in \mathbb{R}_{+}, f(0, I)=f(s, 0)=0$; $\partial f(s, I) / \partial s \geq 0$, for $I>0 ; \partial f(s, I) / \partial s^{2}$ is an increasing function for $s$, while $\partial f$ $(s, I) / \partial I \geq 0$ for $s>0 ; \partial f^{2}(s, I) / \partial I^{2} \leq 0 ; f(s, I) / s$ is a decreasing function with respect to $s$ for $I>0$.

The function $f(s, I)$ satisfies the local Lipschitz continuous in $s$ and $J$, which coincides the assumption in [15]

$$
\begin{aligned}
& \left\|\int_{0}^{\infty} f\left(s_{1}(t, \cdot), I_{1}(t)\right)-f\left(s_{2}(t, \cdot), I_{2}(t)\right)\right\| \\
& \quad \leq L\left(\left\|s_{1}(t, \cdot)-s_{2}(t, \cdot)\right\|_{1}+\left\|I_{1}(t)-I_{2}(t)\right\|_{\mathbb{R}}\right) .
\end{aligned}
$$

Equation $R$ is separated with other equations of system (1), and it can be omitted. Hence, system (1) can lower as a two-dimensional system:

$$
\begin{aligned}
\frac{\partial s(t, a)}{\partial t}+\frac{\partial s(t, a)}{\partial a} & =-\mu s(t, a)-\beta(a) f(s(t, a), I(t)), \\
s(t, 0) & =\Lambda, \\
\frac{d I(t)}{d t} & =\int_{0}^{\infty} \beta(a) f(s(t, a), I(t)) d a-(\mu+\delta) I(t),
\end{aligned}
$$

where $\delta=\gamma+\delta_{1}$. The goal of this paper is to establish global dynamics for (5). This paper supplements or generalizes many existing results $[10,14]$ if we take some special incidences or some specific parameters.

Note that the total population $N(t)=\int_{0}^{\infty} s(t, a) d a+I$ $(t)+R(t)$ satisfies the following equation:

$$
\frac{d N(t)}{d t}=\Lambda-\mu N(t)-\delta I(t) \leq \Lambda-\mu N(t),
$$

which implies that $\limsup _{t \rightarrow \infty} N(t) \leq \Lambda / \mu$.

1.1. Integrated Semigroup Formula. In this subsection, we employ the approach proposed by Thieme [18] to formulate system (5) as an abstract Cauchy problem. Now, we define the state space as follows:

$$
\begin{aligned}
X & :=\mathbb{R} \times L^{1}\left(\mathbb{R}_{+} ; \mathbb{R}\right) \times \mathbb{R}, \\
X_{+} & =\mathbb{R}_{+} \times L_{+}^{1}\left(\mathbb{R}_{+} ; \mathbb{R}_{+}\right) \times \mathbb{R}_{+}, \\
X_{0} & =\{0\} \times L^{1}\left(\mathbb{R}_{+} ; \mathbb{R}\right) \times \mathbb{R}, \\
X_{0,+} & =\{0\} \times L_{+}^{1}\left(\mathbb{R}_{+} ; \mathbb{R}_{+}\right) \times \mathbb{R}_{+},
\end{aligned}
$$

with the norm form given by

$$
\begin{aligned}
\left\|\left(\phi, \psi(\cdot), \psi_{1}(t)\right)\right\| X & =|\phi|+\|\psi(\cdot)\|_{1}+\left|\psi_{1}\right| \\
& =|\phi|+\int_{0}^{\infty} \psi(a) d a+\left|\psi_{1}\right| .
\end{aligned}
$$

In order to formulate system (5) as an abstract Cauchy problem, we define the linear operator $A$ on $X_{0}$ as follows:

$$
A\left(\begin{array}{c}
0 \\
\psi \\
\psi_{1}
\end{array}\right):=\left(\begin{array}{c}
-\psi(0) \\
-\frac{d \psi(a)}{d a}-\mu \psi(a) \\
-(\mu+\delta) \psi_{1}
\end{array}\right),\left(\begin{array}{c}
0 \\
\psi \\
\psi_{1}
\end{array}\right) \in D(A)
$$

where $D(A)=\{0\} \times W^{1,1}(\mathbb{R} ; \mathbb{R}) \times \mathbb{R}$ and $W^{1,1}(\mathbb{R} ; \mathbb{R})$ denote the Sobolev space. The nonlinear operator $F$ on $X_{0}$ is given.

$$
F\left(\begin{array}{c}
0 \\
\psi \\
\psi_{1}
\end{array}\right):=\left(\begin{array}{c}
\Lambda \\
-\beta(a) f\left(\psi(a), \psi_{1}\right) \\
\int_{0}^{\infty} \beta(a) f\left(\psi+(a), \psi_{1}\right) d a
\end{array}\right),\left(\begin{array}{c}
0 \\
\psi \\
\psi_{1}
\end{array}\right) \in D(A) .
$$


Note that $D(A) \subset X_{0}$ and $\overline{D(A)}=X_{0}$. Denote $x(t)=$ $(0, s(t, \cdot), I(t))^{\mathrm{T}}$ and $x_{0}=\left(0, s_{0}(\cdot), I_{0}\right)^{\mathrm{T}}$, we can rewrite system (5) as an abstract Cauchy problem.

$$
\begin{aligned}
& \frac{d x(t)}{d t}=A x(t)+F(x(t)), \quad t \geq 0, \\
& x(0)=x_{0} \in \overline{D(A)} \cap X_{0,+} .
\end{aligned}
$$

Next, we will show the existence and uniqueness of system (11) by the results in [19]. First, we will give the following two lemmas.

Lemma 1.1. There exists a real constant $M \geq 1$ and $\omega \in \mathbb{R}$ such that $(\omega,+\infty) \subset \rho(A)$, and

$$
\left\|(\lambda I-A)^{-n}\right\| \leq \frac{M}{(\lambda-w)^{n}}, \quad \text { for all } n \in \mathbb{N}, \lambda>\omega,
$$

where $\rho(A)$ denotes the resolvent set of $A$ and I denotes the identity operator. Furthermore, for all $\lambda>\omega,(\lambda I-A)^{-1}\left(X_{+}\right)$ $\subset X_{+}$.
Proof 1 . For any $\left(0, \psi, \psi_{1}\right)^{\mathrm{T}} \in X$ and $\lambda>-\mu$, it follows from the definitions of the linear operator $A$ that

$$
(\lambda I-A)^{-1}\left(\begin{array}{c}
\widehat{\phi} \\
\widehat{\psi}(a) \\
\widehat{\psi}_{1}
\end{array}\right)=\left(\begin{array}{c}
0 \\
\psi(a) \\
\psi_{1}
\end{array}\right),
$$

which implies that

$$
\left(\begin{array}{c}
\widehat{\phi} \\
\widehat{\psi}(a) \\
\widehat{\psi}_{1}
\end{array}\right)=\left(\begin{array}{c}
\psi(0) \\
\frac{d}{d a} \psi(a)+\mu \psi(a) \\
(\mu+\delta) \psi_{1}
\end{array}\right) .
$$

We directly calculate and obtain

$$
\begin{aligned}
\psi(a) & =\widehat{\phi} e^{-(\lambda+\mu) a}+\int_{0}^{a} \widehat{\psi}(\eta) e^{-(\lambda+\mu)(a-\eta)} d \eta, \\
\psi_{1} & =\frac{\widehat{\psi}_{1}}{\lambda+\mu+\delta} .
\end{aligned}
$$

So that we have the following estimation.

$$
\begin{aligned}
\left\|(\lambda I-A)^{-1}\left(\begin{array}{c}
\widehat{\phi} \\
\widehat{\psi}(a) \\
\widehat{\psi}_{1}
\end{array}\right)\right\| X=|0|+\int_{0}^{\infty} \psi(a) d a+\left|\psi_{1}\right|=\int_{0}^{\infty} \widehat{\phi} e^{-(\lambda+\mu) a} d a+\int_{0}^{\infty} \int_{0}^{a} \widehat{\psi}(\eta) e^{-(\lambda+\mu)(a-\eta)} d \eta d a+\frac{|\widehat{\phi}|}{|\lambda+\mu+\delta|} \\
\leq \frac{|\widehat{\phi}|}{|\lambda+\mu|}+\frac{\|\widehat{\psi}\|_{1}}{|\lambda+\mu|}+\frac{\|\widehat{\psi}\|}{|\lambda+\mu|}=\frac{1}{\lambda+\mu}\left\|\left(\begin{array}{c}
\widehat{\phi} \\
\widehat{\psi}(a) \\
\widehat{\psi}_{1}
\end{array}\right)\right\|_{X} .
\end{aligned}
$$

Hence, $\omega=-\mu$ and $M=1$ are the values that we are looking for in the presentation of the theorem.

Define $\Upsilon_{L}(0)=\left\{\xi \in X_{0} \mid\|\xi\|_{\Upsilon} \leq L\right\}$. Under Assumption 1.1 , we have the following lemma for the nonlinear operator $F$ on $X_{0}$.

Lemma 1.2. Suppose Assumption 1.1 holds. For all $L>0$, there exists $\Delta(L)>0$ such that

$$
\|F(\xi)-F(\widehat{\xi})\|_{X} \leq \Delta(L)\|\xi-\widehat{\xi}\|_{X}
$$

for any $\xi=\left(0, \psi(a), \psi_{1}\right)^{\mathrm{T}} \in X_{0} \cap \Upsilon_{L}(0)$ and $\widehat{\xi}=\left(0, \widehat{\psi}(a), \widehat{\psi}_{1}\right)^{\mathrm{T}}$ $\in X_{0} \cap \Upsilon_{L}(0)$.

Proof 2. For any $\xi, \widehat{\xi} \in X_{0} \cap \Upsilon_{L}(0)$, we have

$$
\begin{aligned}
\|F(\xi)-F(\widehat{\xi})\| & =\left\|\left(\begin{array}{c}
0 \\
-\beta(a) f\left(\psi(a), \psi_{1}\right)+\beta(a) f\left(\widehat{\psi}(a), \widehat{\psi}_{1}\right) \\
\int_{0}^{\infty} \beta(a) f\left(\psi(a), \psi_{1}\right) d a-\int_{0}^{\infty} \beta(a) f\left(\widehat{\psi}(a), \widehat{\psi}_{1}\right) d a
\end{array}\right)\right\|_{X} \\
& \leq 2 \int_{0}^{\infty} \beta(a)\left|f\left(\psi(a), \psi_{1}\right)-f\left(\widehat{\psi}(a), \widehat{\psi}_{1}\right)\right| d a \leq 2 \bar{\beta} L\left(|\psi-\widehat{\psi}|_{1}+\left|\psi_{1}-\widehat{\psi}_{1}\right|\right)=: \Delta(L)\|\xi-\widehat{\xi}\|_{X},
\end{aligned}
$$


where $\Delta=2 \bar{\beta} L$.

Lemmas 1.1 and 1.2 imply that Assumption 3.1 of [19] holds. It follows from Lemma 3.1 in [19] that the existence and uniqueness of system (11) are given by the following theorem.

Theorem 1.3. There exists a unique semiflow $\Phi: \mathbb{R}_{+} \times$ $X_{0,+} \rightarrow X_{0,+}$ for system (11). For all $t \geq 0 \int_{0}^{t} \Phi(t) x_{0} d s \in D(A)$ and for any $x_{0} \in X_{0,+}$,

$$
\Phi(t) x_{0}=x_{0}+A \int_{0}^{t} \Phi(\eta) x_{0} d \eta+\int_{0}^{t} F[\Phi](\eta) x_{0} d \eta .
$$

In what follows, we will give the regularity of the semiflow $\Phi(t) x_{0}$. By the proof process of Theorem 6.3 in [19], we define the set as

$$
D(B)=\{\varphi \in D(A) \mid A \varphi+F(\varphi) \in \overline{D(A)}\} .
$$

Assumption 1.2. For any $\left(0, \psi(a), \psi_{1}\right) \in X_{0}$, the following conditions hold.

$$
\frac{\partial f\left(\psi(a), \psi_{1}\right)}{\partial \psi(a)} \psi(a) \in L^{1}\left(\mathbb{R}_{+}\right), \frac{\partial f\left(\psi(a), \psi_{1}\right)}{\partial \psi_{1}} \in L^{1}\left(\mathbb{R}_{+}\right) .
$$

Obviously, $D(B) \cap X_{0,+}=\overline{X_{0,+}}$. Based on Assumptions 1.1 and 1.2, the nonlinear operator $F$ on $X_{0}$ is differentiable with respect to $t$, and the differentiation of $F$ at $\widehat{\xi}=\left(0, \widehat{\psi}, \widehat{\psi}_{1}\right)^{\mathrm{T}} \in$ $X_{0}$ is given by

$$
F^{\prime}[\widehat{\xi}]\left(\begin{array}{c}
0 \\
\psi \\
\psi_{1}
\end{array}\right)=\left(\begin{array}{c}
-\beta(a) \frac{\partial f\left(\widehat{\psi}(a), \widehat{\psi}_{1}\right)}{\partial \widehat{\psi}(a)} \psi(a)-\beta(a) \frac{\partial f\left(\widehat{\psi}(a), \widehat{\psi}_{1}\right)}{\partial \widehat{\psi}_{1}} \psi_{1} \\
\int_{0}^{\infty} \beta(a) \frac{\partial f\left(\widehat{\psi}(a), \widehat{\psi}_{1}\right)}{\partial \psi(a)} \psi(a) d a+\int_{0}^{\infty} \beta(a) \frac{\partial f\left(\widehat{\psi}(a), \widehat{\psi}_{1}\right)}{\partial \psi_{1}} \psi_{1} d a
\end{array}\right),\left(\begin{array}{l}
0 \\
\psi \\
\psi
\end{array}\right) \in X_{0} .
$$

From what has been discussed, we obtain the regularity of the semiflow $\Phi(t)$ on $X_{0}$.

Theorem 1.4. For any $x_{0} \in D(B) \cap X_{0,+}$, then the semiflow $\Phi(t) x_{0}$ is continuously differentiable with respect to $t$, and $x$ $(t)=\Phi(t) x_{0}$ is the global classical solution of the abstract Cauchy problem (11).

Based on Assumption 1.1, we have the following priori estimations.

Lemma 1.5. For $(0, s, I)^{\mathrm{T}} \in X_{0,+}$ and $t \geq 0$, the following estimations hold:

$$
\int_{0}^{\infty} s(t, a) d a+I(t) \leq \max \left\{\int_{0}^{\infty} s_{0}(a) d a+I_{0}, \frac{\Lambda}{\mu}\right\}:=M_{1},
$$

and

$$
J(t):=\int_{0}^{\infty} \beta(a) f(s(t, a), I(t)) d a \leq \bar{\beta} \int_{0}^{\infty} f\left(\mu M_{1} \pi(a), M_{1}\right) d a .
$$

\section{Furthermore,}

$$
\limsup _{t \rightarrow \infty}\left(\int_{0}^{\infty} s(t, a) d a+I(t)\right) \leq \frac{\Lambda}{\mu},
$$

and

$$
\limsup _{t \rightarrow \infty} J(t) \leq \bar{\beta} \int_{0}^{\infty} f\left(\Lambda \pi(a), \frac{\Lambda}{\mu}\right) .
$$

Before ending this section, we define a set

$$
\Gamma=\left\{\left(0, s_{0}(\cdot), I_{0}\right)^{\mathrm{T}} \in X_{0}: s_{0}(0)=\Lambda, N(0) \leq \frac{\Lambda}{\mu}\right\} .
$$

Then, it follows from Lemma 1.5 that $\Gamma$ is positively invariant. This indicates that $\Phi\left(t, x_{0}\right) \in \Gamma, \forall t \geq 0$, and $x_{0}$ $\in \Gamma$. In what follows, for convenience, we denote $(0, s, I)^{\mathrm{T}}$ as $(s, I)^{\mathrm{T}}$.

The paper is devoted to study the global stability of the steady states of the system by employing the Lyapunov functionals. The rest of this paper is organized as follows: Section 2 gives the asymptotic smoothness of the solution semiflow $\Phi(t) x_{0}$. In Section 3, we calculate the basic reproduction number by renewal equation and discuss the existence and local stability of steady states of system (5). In Section 4, the global dynamics of system (5) is established by considering the persistence of system (5) and constructing suitable Lyapunov functionals. 


\section{Asymptotic Smoothness}

In view of system (5), we note that it has one integrate term. Hence, it is necessary to investigate the existence of a compact attractor of all bounded sets of $X$. In order to establish this goal, we need the results of Theorem 2.33 and Theorem 2.46 in [20]. To apply them, we decompose the solution semiflow $\Phi=\widehat{\Phi}+\tilde{\Phi}$, where

$$
\begin{aligned}
& \widehat{\Phi}\left(t,\left(s_{0}(\cdot), I_{0}\right)\right)=(\widehat{s}(t, \cdot), 0), \\
& \tilde{\Phi}\left(t,\left(s_{0}(\cdot), I_{0}\right)\right)=(\tilde{s}(t, \cdot), I(t)),
\end{aligned}
$$

where

$$
\tilde{s}(t, a)=\left\{\begin{array}{ll}
s(t, a), & \text { for } 0 \leq a \leq t \\
0, & \text { for } t \leq a
\end{array}= \begin{cases}\Lambda \pi(a)-\int_{0}^{a} \beta(a-\eta) f(s(t-\eta, a-\eta), I(t-\eta)) e^{-\mu \eta} d \eta, & \text { for } 0 \leq a \leq t \\
0, & \text { for } t \leq a\end{cases}\right.
$$

and

$$
\widehat{s}(t, a)=s(t, a)-\tilde{s}(t, a)= \begin{cases}0, & \text { for } 0 \leq a \leq t, \\ s_{0}(a-t) e^{-\mu t}-\int_{0}^{t} \beta(a-\eta) f(s(t-\eta, a-\eta), I(t-\eta)) e^{-\mu t} d \eta, & \text { for } t \leq a .\end{cases}
$$

Theorem 2.1. The semiflow $\Phi: \mathscr{R}_{+} \times X_{0} \rightarrow X_{0}$ is asymptotically smooth if there are two maps $\widehat{\Phi}$ and $\tilde{\Phi}: \mathbb{R}_{+} \times X_{0} \rightarrow X_{0}$ such that $\Phi(t) x_{0}=\widehat{\Phi}(t) x_{0}+\tilde{\Phi}(t) x_{0}$, and for any bounded set $K$, the following statements hold:

(1) For any $x_{0} \in K$, there exists a function $\delta: \mathbb{R}_{+} \times \mathbb{R}_{+}$ $\rightarrow \mathbb{R}_{+}$such that for any $r>0 \lim _{t \rightarrow+\infty} \delta(t, r)=0$ with $\left\|x_{0}\right\|_{K} \leq r$, then $\left\|\widehat{\Phi}(t) x_{0}\right\| \leq \delta(t, r)$.

(2) There exists a $t_{K}>0$ such that $\tilde{\Phi}(t) x_{0}$ has a compact closure for each $t \geq t_{K}$.
Lemma 2.2. For any $r>0$, there exists a function $\delta: \mathbb{R}_{+} \times \mathbb{R}_{+}$ such that for any $r>0$,

$$
\lim _{t \rightarrow+\infty} \delta(t, r)=0,
$$

and

$$
\left\|\widehat{\Phi}(t) x_{0}\right\|_{K} \leq \delta(t, r), \quad \forall x_{0} \in K, t \geq 0 .
$$

Proof 3. This lemma follows the process as Theorem 2.46 in [21]. Let $K$ be a bounded subset of $\left(s_{0}(\cdot), I_{0}\right) \in K \subset \Gamma$. It is easy to see that $\widehat{s}, \widehat{I}$ and $\tilde{s}, \tilde{I}$ are nonnegative. Using (30) leads to

$$
\begin{aligned}
\left\|\widehat{\Phi}\left(t,\left(s_{0}(\cdot), I_{0}\right)\right)\right\|_{K} & =\|\widehat{s}(t, \cdot)\|_{1}=\int_{t}^{\infty} s_{0}(a-t) e^{-\mu t} d a-\int_{t}^{\infty} \int_{0}^{a} \beta(a-\eta) f(s(a-\eta, t-\eta), I(t-\eta)) e^{-\mu \eta} d \eta d a \leq e^{-\mu t} \int_{t}^{\infty} s_{0}(a-t) d a \\
& =e^{-\mu t} \int_{0}^{\infty} s_{0}(a) d a=e^{-\mu t}\left\|s_{0}\right\|_{1}=e^{-\mu t}\left\|\left(s_{0}(\cdot), I_{0}\right)\right\|_{K} \leq \delta(t, r),
\end{aligned}
$$

where $\delta(t, r)=e^{-\mu t} r$. Hence, the assumption in (1) of Theorem 2.1 holds.

Next, we prove that condition (2) of Theorem 2.1 is also satisfied. To achieve such goal, we employ the FréchetKolmogorov theorem for the compactness of sets in $L^{1}$ [21].
Theorem 2.3 (Fréchet-Kolmogorov theorem, [21]).

Let $K$ be a subset of $L^{1}\left(R_{+}\right)$. Then, $K$ has compact closure if and only if the following conditions hold:

(i) $\sup _{f \in K} \int_{0}^{\infty}|f(a)| d a<\infty$, 
(ii) $\lim _{r \rightarrow \infty} \int_{r}^{\infty}|f(a)| d a=0$, uniformly in $f \in K$,

(iii) $\lim _{h \rightarrow 0^{+}} \int_{0}^{\infty}|f(a+h)-f(a)| d a=0$, uniformly in $f \in K$,

(iv) $\lim _{h \rightarrow 0^{+}} \int_{0}^{h}|f(a)| d a=0$, uniformly in $f \in K$.

Lemma 2.4. $\tilde{\Phi}(t)$ maps any bounded subsets of $K$ into sets with compact closure in $X_{0}$.

Proof 4. For any fixed $t \in \mathbb{R}_{+}$and any bounded set $K \subseteq \Gamma_{0}$, the set $K_{t} \triangleq\left\{\tilde{\Phi}\left(t,\left(s_{0}, I_{0}\right)\right):\left(s_{0}, I_{0}\right) \in K\right\}$ is precompact. With a notice, $I(t)$ is bounded by the virtue of $f(x, y)$ and Lemma 1.5. This implies that $K_{t, s}=\left\{\tilde{s}(t):(\tilde{s}(t, \cdot), I(t)) \in K_{t}\right\}$ is precompact. It is enough to show that $K_{t, s}=\{\tilde{s}(t, \cdot):(\tilde{s}(t, \cdot), I$ $\left.(t)) \in K_{t}\right\}$ is precompact. This can be achieved by applying Fréchet-Kolmogorov Theorem 2.3 in [21]. First, $s(t, a) \leq$
$\Lambda \pi(a)$ and $\int_{0}^{\infty} \pi(a) d a=1 / \mu$ indicate that $K_{t, s}$ is bounded. This implies that condition (i) of Fréchet-Kolmogorov theorem holds. Second, it is an immediate result that $\int_{r}^{\infty} \tilde{s}(t, a) d a$ approaches zero as $r$ goes to infinity. By the boundedness of $s(t, a)$, condition (iv) of Fréchet-Kolmogorov theorem is well established. Finally, condition (iii) of the FréchetKolmogorov theorem should be verified. In order to prove it, we should show that $\tilde{\Phi}$ is uniformly continuous on the bounded set $K_{t, s}$ or

$$
\lim _{h \rightarrow 0^{+}}\|\tilde{s}(t, \cdot)-\tilde{s}(t, \cdot+h)\|_{1}=0 \text {, uniformly in } K_{t, s} .
$$

Equation (34) holds obviously when $t=0$ since $\tilde{s}(0, \cdot)=$ $s_{0}(a)$. So, we need to show that the result holds for $t>0$. Since we are concerned with the limit as $h$ tends to $0^{+}$, we assume that $h \in(0, t)$. Then,

$$
\begin{aligned}
\|\tilde{s}(t, \cdot)-\tilde{s}(t, \cdot h)\|_{1}= & \int_{0}^{\infty}|\tilde{s}(t, a)-\tilde{s}(t, a+h)| d a \\
= & \int_{0}^{t-h}\left|\int_{0}^{a+h} \beta(a+h-\eta) f(s(t-\eta, a+h-\eta), I(t-\eta)) \pi(\eta)-\int_{0}^{a} \beta(a-\eta) f(s(t-\eta, a-\eta), I(t-\eta)) \pi(\eta)\right| d \eta d a \\
& +\int_{t-h}^{t}\left|\int_{0}^{a} \beta(a-\eta) f(s(t-\eta, a-\eta), I(t-\eta)) \pi(\eta) d \eta\right| d a+\Lambda \int_{0}^{\infty}|\pi(a+h)-\pi(a)| d a \\
\leq & \int_{0}^{t-h} \int_{a}^{a+h} \beta(a+h-\eta) f(s(t-\eta, a+h-\eta), I(t-\eta) \pi(\eta) d \eta d a \\
& +\int_{0}^{t-h} \int_{0}^{a}|\beta(a+h-\eta)-\beta(a-\eta)| f(s(t-\eta, a+h-\eta), I(t-\eta) \pi(\eta) d \eta d a \\
& +\int_{0}^{t-h} \int_{0}^{a} \beta(a-\eta) \mid f(s(t-\eta, a+h-\eta), I(t-\eta)-f(s(t-\eta, a-\eta), I(t-\eta) \mid \pi(\eta) d \eta d a \\
& +\bar{\beta} f\left(\Lambda, \frac{\Lambda}{\mu}\right) h+\Lambda(t-h) h \\
\leq & f\left(\Lambda, \frac{\Lambda}{\mu}\right)(t-h) h+f\left(\Lambda, \frac{\Lambda}{\mu}\right)\left(1+\frac{1}{\mu}\right)(t-h) L \beta+2 \Lambda(t-h) h+\bar{\beta} f\left(\Lambda, \frac{\Lambda}{\mu}\right) h+\Lambda(t-h) h \\
\leq & \left\{\left[f\left(\Lambda, \frac{\Lambda}{\mu}\right)\left(1+L_{\beta}\left(1+\frac{1}{\mu}\right)\right)+3 \Lambda\right](t-h)+\bar{\beta} f\left(\Lambda, \frac{\Lambda}{\mu}\right)\right\} h,
\end{aligned}
$$

since $s(t, a) \leq \Lambda \pi(a), I(t) \leq \Lambda / \mu$ for $t, a \in \mathbb{R}_{+}$, and $\mid \pi(a+h)-$ $\pi(a) \mid \leq 1-e^{-\int_{a}^{a+h} \mu s d s} \leq \mu h$. This estimate immediately yields (34).

With the help of Lemmas 2.2 and 2.4, together with $\left\|\Phi(t) x_{0}\right\|_{X} \leq \Lambda / \mu$, we conclude that $\Phi(t)$ has compact closure in $X$ for any $x_{0} \in K$. Theorem 2.1 ensures that the semiflow $\Phi(t)$ is asymptotically smooth.

Proposition 2.5. Let Assumption 1.1 hold, and then, the solution semiflow $\Phi$ defined by (19) on $\Gamma_{0}$ is asymptotically smooth.

\section{The Basic Reproduction Number, Existence, and Local Stability of Steady States}

In this section, we give the general approach for calculating the basic reproduction number, which is the spectral radius of the next-generation operator. The basic reproduction number is the most important value for characterizing the renewal process in structured population. An epochmaking method was proposed by Diekmann et al. in [22] and then developed by Inaba in [23]. Moreover, Yang and $\mathrm{Xu}$ in [24] extend this method to calculate the basic reproduction of disease models on complex networks. Let $(\bar{s}(\cdot), \bar{I})$ 
be a solution of system (5), and then, it satisfies the following equations:

$$
\begin{aligned}
\frac{d \bar{s}(a)}{d a} & =-\mu \bar{s}(a)-\beta(a) f(\bar{s}(a), \bar{I}), \\
\bar{s}(0) & =\Lambda, \\
0 & =\int_{0}^{\infty} \beta(a) f(\bar{s}(a), \bar{I}) d a-(\mu+\delta) \bar{I} .
\end{aligned}
$$

Obviously, system (36) has a unique disease-free steady state $E_{0}=(\Lambda \pi(a), 0)$. Now, we are in position to define the basic reproduction number by analyzing the renewal equation. The linear equation in disease-free invasion phase is given by

$$
\frac{d I(t)}{d t}=\int_{0}^{\infty} \beta(a) \frac{\partial f(\Lambda \pi(a), 0)}{\partial I} d a I(t)-(\mu+\delta) I(t) .
$$

Solving (37) yields

$$
\begin{aligned}
I(t) & =I_{0} e^{-(\mu+\delta) t}+\int_{0}^{\infty} \beta(a) \frac{\partial f(\Lambda \pi(a), 0)}{\partial I} d a \int_{0}^{t} I(s) e^{-(\mu+\delta)(t-s)} d s \\
& =I_{0} e^{-(\mu+\delta) t}+\int_{0}^{\infty} \beta(a) \frac{\partial f(\Lambda \pi(a), 0)}{\partial I} d a \int_{0}^{t} I(t-s) e^{-(\mu+\delta) s} d s .
\end{aligned}
$$

Hence, the basic reproduction number is calculated as

$$
\begin{aligned}
\mathscr{R}_{0} & =\int_{0}^{\infty} \beta(a) \frac{\partial f(\Lambda \pi(a), 0)}{\partial I} d a \int_{0}^{\infty} e^{-(\mu+\delta) s} d s \\
& =\frac{1}{\mu+\delta} \int_{0}^{\infty} \beta(a) \frac{\partial f(\Lambda \pi(a), 0)}{\partial I} d a .
\end{aligned}
$$

In epidemiology, the basic reproduction number $\mathscr{R}_{0}$ gives the average number of cases that a typical infectious individual generates, if introduced into a susceptible population, over the whole infectious period.

Next, we look for the solution $(\bar{s}(a), \bar{I}) /\left\{E_{0}\right\}$. It follows from the third equation of (36) that

$$
(\mu+\delta) \bar{I}=\int_{0}^{\infty} \beta(a) f(\bar{s}(a), \bar{I}) d a .
$$

Solving the first equation of system (36), we have

$$
\bar{s}(a)=\Lambda \pi(a)-\int_{0}^{a} \beta(\tau) f(\bar{s}(\tau), \bar{I}) d \tau .
$$

Then, we integrate (41) to obtain

$$
\begin{aligned}
\bar{S} & :=\int_{0}^{\infty} \bar{s}(a) d a=\frac{\Lambda}{\mu}-\int_{0}^{\infty} \int_{0}^{a} \beta(\tau) f(s(\tau), \bar{I}) \frac{\pi(a)}{\pi(\tau)} d \tau d a \\
& =\frac{\Lambda}{\mu}-\int_{0}^{\infty} \beta(\tau) f(\bar{s}(\tau), \bar{I}) e^{\mu \tau \tau} \int_{s}^{\infty} e^{-\mu a} d a d \tau \\
& =\frac{\Lambda}{\mu}-\frac{1}{\mu} \int_{0}^{\infty} \beta(a) f(\bar{s}(a), \bar{I}) d a .
\end{aligned}
$$

Moreover, we replace $\int_{0}^{\infty} \beta(a) f(\bar{s}(a), \bar{I}) d a$ in (42) by (40) and obtain

$$
\bar{I}=\frac{\mu}{\mu+\delta}\left(\frac{\Lambda}{\mu}-\bar{S}\right) .
$$

Combining (40) and (43), we have

$$
\begin{gathered}
\bar{I}=\frac{\mu}{\mu+\delta}\left(\frac{\Lambda}{\mu}-\bar{S}\right), \\
(\mu+\delta) \bar{I}=\int_{0}^{\infty} \beta(a) f(\bar{s}(a), \bar{I}) d a .
\end{gathered}
$$

Borrowing the first equation of system (44), we obtain the following equation consisting of one variable

$$
g(\bar{S}):=\int_{0}^{\infty} \beta(a) f\left(\bar{s}(a), \frac{\mu}{\mu+\delta}\left(\frac{\Lambda}{\mu}-\bar{S}\right)\right) d a-\mu\left(\frac{\Lambda}{\mu}-\bar{S}\right)=0 .
$$

With a notice,

$$
\begin{aligned}
g^{\prime}(\bar{S}):=\int_{0}^{\infty} \beta(a)[ & \frac{\partial f(\bar{s}(a), \mu /(\mu+\delta)(\Lambda / \mu-\bar{S}))}{\partial \bar{s}} \\
& \left.-\frac{\mu}{\mu+\delta} \frac{\partial f(\bar{s}(a), \mu /(\mu+\delta)(\Lambda / \mu-\bar{S}))}{\partial \bar{I}}\right] d a+\mu=0 .
\end{aligned}
$$

Then $g^{\prime}(\Lambda / \mu)=\mu\left(1-\mathscr{R}_{0}\right)<0$ if $\mathscr{R}_{0}>1$. Besides, we note that $g(\Lambda / \mu)=0$ and $g(0)=-\Lambda<0$. Therefore, there exists at least one positive solution of (45) for $\bar{S} \in(0, \Lambda / \mu)$.

Next, we will show the uniqueness of the endemic steady state of system (44). By way of contradiction, we assume that there exist two positive solutions of $g(x)=0$, denoted by $\bar{s}$ and $\widehat{s}$, respectively. They satisfy that $\bar{s}(a)>\widehat{s}$ (a). It follows from the relations between $\bar{s}(a)$ and $\bar{I}$ that $\bar{I}<\widehat{I}$, that is, $\widehat{I}=q \bar{I}$ with $q>1$. By the second equation of (44) and the property of $f(x, y)$ with respect to $x$, we obtain

$$
\begin{aligned}
\widehat{I} & =\frac{1}{\mu+\delta} \int_{0}^{\infty} \beta(a) f(\widehat{s}(a), \widehat{I}) d a \\
& \leq \frac{1}{\mu+\delta} \int_{0}^{\infty} \beta(a) f(\bar{s}(a), \widehat{I}) d a \\
& =\frac{1}{\mu+\delta} \int_{0}^{\infty} \beta(a) f(\bar{s}(a), q \bar{I}) d a \\
& <\frac{1}{\mu+\delta} \int_{0}^{\infty} \beta(a) \frac{f(\bar{s}(a), \bar{I})}{\bar{I}} q \bar{I} d a=q \bar{I} .
\end{aligned}
$$

This is a contradiction with assumption $q \bar{I}=\widehat{I}$. From what has been discussed, we have the following result concerning the existence of steady states.

Theorem 3.1. Consider system (5) with $\mathscr{R}_{0}$ defined in (39). If $\mathscr{R}_{0} \leq 1$, then there is a unique steady state, which is the disease-free steady state $E_{0}$; while if $\mathscr{R}_{0} \leq 1$, then there are two steady states, the disease-free steady state $E_{0}$ and the endemic steady state $E^{*}$.

Secondly, we study the local stability of the steady states obtained in Theorem 3.1. In order to do this, we linearize system (5) at the endemic steady state $E^{*}$ and obtain 


$$
\begin{aligned}
\frac{\partial x(t, a)}{\partial t}+\frac{\partial x(t, a)}{\partial a}= & -\mu x(t)-\beta(a) \frac{\partial f(\bar{s}(a), \bar{I})}{\partial \bar{s}} x(t, a) \\
& -\beta(a) \frac{\partial f(\bar{s}(a), \bar{I})}{\partial \bar{I}} y(t), \\
x(t, 0)= & 0, \\
\frac{d y(t)}{d t}= & \int_{0}^{\infty} \beta(a) \frac{\partial f(\bar{s}(a), \bar{I})}{\partial \bar{s}(a)} x(t, a) d a \\
& +\int_{0}^{\infty} \beta(a) \frac{\partial f(\bar{s}(a), \bar{I})}{\partial \bar{I}} \operatorname{day}(t) \\
& -(\mu+\delta) y(t) .
\end{aligned}
$$

Now, we focus on system (48) and obtain the local stability of the steady states.

Theorem 3.2. Let $\mathscr{R}_{0}$ define in (39). If $\mathscr{R}_{0}<1$, then the disease-free steady state $E_{0}$ is locally asymptotically stable and if $\mathscr{R}_{0}<1$, the unique endemic steady state $E^{*}$ is locally asymptotically stable.

Proof 5. Let $x(t, a)=x(a) e^{\lambda t}$ and $y(t)=y_{0} e^{\lambda t}$ be a solution of (48). Then, system (48) can be formally written as

$$
\begin{aligned}
\frac{d x(a)}{d a}= & -(\lambda+\mu) x(a)-\beta(a) \frac{\partial f(\bar{s}(a), \bar{I})}{\partial \bar{s}(a)} x(a) \\
& -\beta(a) \frac{\partial f(\bar{s}(a), \bar{I})}{\partial \bar{I}} y_{0}, \\
x(0)= & 0, \\
0= & \int_{0}^{\infty} \beta(a) \frac{\partial f(\bar{s}(a), \bar{I})}{\partial \bar{s}(a)} x(a) d a \\
& +\int_{0}^{\infty} \beta(a) \frac{\partial f(\bar{s}(a), \bar{I})}{\partial \bar{I}} d a y_{0}-(\lambda+\mu+\delta) y_{0} .
\end{aligned}
$$

Solving the first equation yields

$$
x(a)=-y_{0} \int_{0}^{a} \beta(\tau) \frac{\partial f(\bar{s}(\tau), \bar{I})}{\partial \bar{I}} e^{-\int_{\tau}^{a}(\lambda+\mu+\beta(\xi)((\partial f(\bar{s}(\xi), \bar{I})) / \partial \bar{s})) d \xi} d \tau
$$

Then substituting (50) into the equation $z:=\int_{0}^{\infty} \beta(a) \partial$ $f(\bar{s}(a), \bar{I}) / \partial \bar{s}(a) x(a) d a$, we have

$$
z=-y_{0} \int_{0}^{\infty} \beta(a) \frac{\partial f(\bar{s}(a), \bar{I})}{\partial \bar{s}} \int_{0}^{\infty} \beta(\tau) \frac{\partial f(\bar{s}(\tau), \bar{I})}{\partial \bar{I}} e^{-\int_{\tau}^{a}(\lambda+\mu \mid+\beta(\xi)((\partial f(\bar{s}(\xi), \bar{I})) / \partial \bar{s})) d \xi} d \tau d a
$$

After variable change and integration by parts, we obtain

$$
\begin{aligned}
z & =-y_{0} \int_{0}^{\infty} \beta(\tau) \frac{\partial f(\bar{s}(\tau), \bar{I})}{\partial \bar{I}} \int_{0}^{\infty} \beta(a) \frac{\partial f(\bar{s}(a), \bar{I})}{\partial \bar{s}} e^{-\int_{\tau}^{a}(\lambda+\mu \mid+\beta(\xi)((\partial f(\bar{s}(\xi), \bar{I})) / \partial \bar{s})) d \xi} d a d \tau \\
& =y_{0} \int_{0}^{\infty} \beta(\tau) \frac{\partial f(\bar{s}(\tau), \bar{I})}{\partial \bar{I}}\left(\left.e^{-\int_{\tau}^{a}(\lambda+\mu \mid+\beta(\xi)((\partial f(\bar{s}(\xi), \bar{I})) / \partial \bar{s})) d \xi}\right|_{\tau} ^{\infty}+(\lambda+\mu) \int_{t}^{\infty} e^{-\int_{\tau}^{a}(\lambda+\mu \mid+\beta(\xi)((\partial f(\bar{s}(\xi), \bar{I})) / \partial \bar{s})) d \xi} d a\right) d \tau \\
& =y_{0} \int_{0}^{\infty} \beta(\tau) \frac{\partial f(\bar{s}(\tau), \bar{I})}{\partial \bar{I}}\left(-1+(\lambda+\mu) \int_{0}^{\infty} e^{-\int_{\tau}^{a}(\lambda+\mu \mid+\beta(\xi)((\partial f(\bar{s}(\xi), \bar{I})) / \partial \bar{s})) d \xi} d a\right) d \tau
\end{aligned}
$$

Plugging (53) into the second equation of (49), we admit

$$
\begin{aligned}
& y_{0}(\lambda+\mu) \int_{0}^{\infty} \beta(\tau) \frac{\partial f(\bar{s}(\tau), \bar{I})}{\partial \bar{I}} \int_{\tau}^{\infty} e^{-\int_{\tau}^{a}(\lambda+\mu+\beta(\xi)((\partial f(\bar{s}(\xi), \bar{I})) / \partial \bar{s})) d \xi} d a d \tau \\
& \quad=y_{0}(\lambda+\mu+\delta) .
\end{aligned}
$$

Equation (53) is the characteristic equation of (5). Then, the steady state $\bar{E}$ is locally (asymptotically) stable if all eigenvalues of the characteristic equation have negative real parts and it is unstable if at least one eigenvalue has a positive real part.
Firstly, we note that the characteristic equation at $E_{0}$ is

$$
(\lambda+\mu) \int_{0}^{\infty} \beta(\tau) \frac{\partial f\left(s^{0}(\tau), 0\right)}{\partial \bar{I}} \int_{\tau}^{\infty} e^{-\int_{\tau}^{a}(\lambda+\mu) d \xi} d a d \tau=(\lambda+\mu+\delta) .
$$

Integrating the inner formula yields

$$
(\lambda+\mu+\delta) \int_{0}^{\infty} \beta(a) \frac{\partial f(\Lambda \pi(a), 0)}{\partial I} e^{-\int_{0}^{a}(\lambda+\mu) d \xi} d a .
$$

We claim that all roots of (55) have negative real parts. Otherwise, let $\lambda_{0}$ be a root of (55) with $\operatorname{Re}\left(\lambda_{0}\right) \geq 0$. 
Then, the module of the left hand side of (55) is larger than $\mu+\delta$, while the module of the right hand side of (55) is $\left|\int_{0}^{\infty} \beta(a) \partial f(\Lambda \pi(a), 0) / \partial I e^{-\int_{0}^{a}(\lambda+\mu) d \xi} d a\right| \leq \mathscr{R}_{0}(\mu+\delta)$. If $\mathscr{R}_{0}<1$, this leads to a contradiction. Therefore, all the eigenvalues of the characteristic (54) have negative real part and then disease-free steady state $E_{0}$ is locally asymptotically stable if $\mathscr{R}_{0}<1$.

Secondly, by the virtue of $f$, we readily obtain

$$
\begin{aligned}
\mid & (\lambda+\mu) \int_{0}^{\infty} \beta(\tau) \frac{\partial f(\bar{s}(\tau), \bar{I})}{\partial \bar{I}} \int_{\tau}^{\infty} e^{-\int_{\tau}^{a}(\lambda+\mu+\beta(\xi)((\partial f(\bar{s}(\bar{\xi}) \bar{I})) / \partial \bar{s})) d \xi} d a d \tau \mid \\
& \leq\left|(\lambda+\mu) \int_{0}^{\infty} \beta(\tau) \frac{f(\bar{s}(\tau), \bar{I})}{\bar{I}} \int_{\tau}^{\infty} e^{-\int_{\tau}^{a}(\lambda+\mu) d \xi} d a d \tau\right| \\
& =\left|\int_{0}^{\infty} \beta(\tau) \frac{f(\bar{s}(\tau), \bar{I})}{\bar{I}} e^{-\int_{0}^{\tau}(\lambda+\mu) d \xi} d a d \tau\right| .
\end{aligned}
$$

Next, we show that the characteristic equation (53) has no eigenvalues with nonnegative real parts. Arguing directly, assume that there is one eigenvalue $\lambda_{1}$ with $\operatorname{Re}\left(\lambda_{1}\right) \geq 0$. Then

$$
\begin{aligned}
& \left|\int_{0}^{\infty} \beta(\tau) \frac{f(\bar{s}(\tau), \bar{I})}{\bar{I}} e^{-\int_{0}^{\tau}(\lambda+\mu) d \xi} d a d \tau\right| \\
& \quad \leq\left|\int_{0}^{\infty} \beta(\tau) \frac{f(\bar{s}(\tau), \bar{I})}{\bar{I}} \pi(\tau) d \tau\right|<\mu+\delta .
\end{aligned}
$$

On the other hand, by the right hand side of (53), we have

$$
|\lambda+\mu+\delta|>\mu+\delta
$$

Consequently, the endemic steady state $E^{*}$ is locally asymptotically stable.

\section{Global Stability Analysis}

In order to consider the compactness of the orbit $\Phi\left(t, s_{0}(\cdot)\right.$, $\left.\left.I_{0}\right)\right) \in \Gamma$, we use the invariance principle which is referred in Theorem 4.2 of Chapter IV [21]. Therefore, we can define $\rho: \Gamma \rightarrow \mathbb{R}_{+}$as

$$
\rho(s(t, \cdot), I(t))=I(t), \quad \text { for }(s(t, \cdot), I(t)) \in \Gamma .
$$

Let

$$
\begin{aligned}
& \Gamma_{0}=\left\{\left(s_{0}(\cdot), I_{0}\right) \in \Gamma \text { : there exists a } t_{0}\right. \\
&\left.\in \mathbb{R}_{+} \text {such that } \rho\left(\Phi\left(t_{0},\left(s_{0}(\cdot), I_{0}\right)\right)\right)>0\right\} .
\end{aligned}
$$

Obviously, if $\left(s_{0}(\cdot), I_{0}\right) \in \Gamma / \Gamma_{0}$, then $(s(t, \cdot), I(t)) \rightarrow E_{0}$ as $t \rightarrow \infty$.

\section{Definition 4.1 ([21], page 61).}

If there exists an $\epsilon>0$, independent of the initial conditions, such that

$$
\limsup _{t \rightarrow \infty} \rho\left(\Phi\left(t,\left(s_{0}(\cdot), I_{0}\right)\right)\right)>\epsilon\left(\operatorname{resp} ., \liminf _{t \rightarrow \infty} \rho\left(\Phi\left(t,\left(s_{0}(\cdot), I_{0}\right)\right)\right)>\epsilon\right),
$$

then (5) is called to be uniformly weakly $\rho$-persistent (resp., uniformly strongly $\rho$-persistent), for $\left(s_{0}(\cdot), I_{0}\right) \in \Gamma_{0}$.

To achieve the persistence of the system (5), we firstly prove the weekly $\rho$-persistence and then give the global compactness of the orbit.

Proposition 4.2. If $\mathscr{R}_{0}>1$, then (5) is uniformly weakly $\rho$ persistent.

Proof 6. Suppose $\mathscr{R}_{0}>1$, there exists an $\epsilon^{0}>0$ such that

$$
\frac{1}{\mu+\delta} \int_{0}^{\infty} \beta(a)\left(\frac{\partial f\left(\Lambda \pi(a)-\int_{0}^{a} \beta(s) f\left(\Lambda \pi(s), \epsilon_{0}\right) \pi(s) d s, \epsilon^{0}\right)}{\partial I}\right) e^{-\lambda a} d a>1 .
$$

Arguing directly, there exists $\left(s_{0}(\cdot), I_{0}\right) \in \Gamma_{0}$ with

$$
\limsup _{t \rightarrow \infty} \rho\left(\Phi\left(t,\left(s_{0}(\cdot), I_{0}\right)\right)\right) \leq \frac{\epsilon^{0}}{2} .
$$

Then, there exists $t_{0} \in \mathbb{R}_{+}$such that

$$
\rho\left(\Phi\left(t,\left(s_{0}(\cdot), I_{0}\right)\right)\right) \leq \epsilon^{0}, \quad \text { for } t \geq t_{0} .
$$

Without loss of generality, we can assume that $t_{0}=0$ since we can achieve it by replacing the initial condition with $\Phi\left(t_{0},\left(s_{0}(\cdot), I_{0}\right)\right)$. Then, for $t \geq t_{0}=0$ with a notice $s(t, a) \leq$ $\Lambda \pi(a)$ and (A3) of Assumption 1.1 for the function $f(s(t, a)$, $I(t))$ with respect to variables $s$ and $I$, we have

$$
\frac{\partial s(t, a)}{\partial a}+\frac{\partial s(t, a)}{\partial a} \geq-\mu s(t, a)-\beta(a) f\left(\Lambda \pi(a), \epsilon_{0}\right),
$$

and this implies that

$$
s(t, a)=\left\{\begin{array}{l}
\Lambda \pi(a)-\int_{0}^{a} \beta(a-\eta) f\left(\Lambda \pi(a-\eta), \epsilon_{0}\right) \pi(\eta) d \eta, \quad t \geq a, \\
s_{0}(a-t) \pi(t)-\int_{0}^{t} \beta(a-\eta) f\left(\Lambda \pi(a-\eta), \epsilon_{0}\right) \pi(\eta) d \eta, \quad t<a .
\end{array}\right.
$$

Therefore,

$$
\begin{aligned}
J(t) & :=\int_{0}^{\infty} \beta(a) f(s(t, a), I(t)) d a \\
& \geq \int_{0}^{t} \beta(a) f\left(\Lambda \pi(a)-\int_{0}^{a} \beta(s) f\left(\Lambda \pi(s), \epsilon_{0}\right) \pi(s) d s, I(t)\right) d a .
\end{aligned}
$$

Trying to consider the third equation of (5), we have 


$$
\begin{aligned}
\dot{I}(t) & =J(t)-(\mu+\delta) I(t) \\
& \geq \int_{0}^{t} \beta(a) f\left(\Lambda \pi(a)-\int_{0}^{a} \beta(a-\eta) f\left(\Lambda \pi(a-\eta), \epsilon_{0}\right) \pi(\eta) d \eta, I(t)\right) d a-(\mu+\delta) I(t) \\
& \left.\geq \int_{0}^{t} \beta(a) \frac{\partial f\left(\Lambda \pi(a)-\int_{0}^{a} \beta(a-\eta) f\left(\Lambda \pi(a-\eta), \epsilon_{0}\right) \pi(\eta) d \eta, \epsilon^{0}\right)}{\partial I}\right) d a I(t)-(\mu+\delta) I(t),
\end{aligned}
$$

where we follow the mean value theorem that

$$
J(t) \geq \int_{0}^{t} \beta(a)\left(\frac{\partial f\left(\Lambda \pi(a)-\int_{0}^{a} \beta(a-\eta) f\left(\Lambda \pi(a-\eta), \epsilon_{0}\right) \pi(\eta) d \eta, \epsilon^{0}\right)}{\partial I}\right) d a I(t),
$$

for $0 \leq I(t) \leq \epsilon^{0}$. Taking Laplace transform on both side of (68) yields

$$
\begin{aligned}
\widehat{I(\lambda)} & \geq \frac{1}{\lambda+\mu+\delta} \int_{0}^{\infty} \beta(a)\left(\frac{\partial f\left(\Lambda \pi(a)-\int_{0}^{a} \beta(a-\eta) f\left(\Lambda \pi(a-\eta), \epsilon_{0}\right) \pi(\eta) d \eta, \epsilon^{0}\right)}{\partial I}\right) e^{-\lambda a} d a \widehat{I(\lambda)}+\frac{I(0)}{\lambda+\mu+\delta} \\
& \geq \frac{1}{\lambda+\mu+\delta} \int_{0}^{\infty} \beta(a)\left(\frac{\partial f\left(\Lambda \pi(a)-\int_{0}^{a} \beta(a-\eta) f\left(\Lambda \pi(a-\eta), \epsilon_{0}\right) \pi(\eta) d \eta, \epsilon^{0}\right)}{\partial I}\right) e^{-\lambda a} d a \widehat{I(\lambda)} .
\end{aligned}
$$

By the arbitrary of $\epsilon^{0}$ and $\lambda$, we take $\epsilon^{0}$ and $\lambda$ which are small enough. Then, (62) contradicts $\mathscr{R}_{0}>1$.

A total trajectory of the semiflow $\Phi$ is a function $g: \mathbb{R}$ $\rightarrow \mathbb{R}_{+} \times L_{+}^{1} \times \mathbb{R}_{+}$such that $\Phi(s, g(t))=g(t+s)$ for all $t \in \mathbb{R}$ and all $s \in \mathbb{R}_{+}$. For the definition of a total trajectory, the solution $(s(t, a), I(t))$ satisfies the following property for $t \in \mathbb{R}$ and $a \in \mathbb{R}_{+}$.

Proposition 4.3. Let $g(t)$ be a total trajectory in $\Gamma$. Then, $s(t, a)$ is positive and $I(t)$ is positive or identically zero for all $t \in \mathbb{R}$.

Proof 7. First, we show that $s(t, a)$ is strictly positive for all $t \in \mathbb{R}$ and $a \in \mathbb{R}_{+}$. From $s$ equation of system (5), we obtain that

$$
\frac{\partial s(t, a)}{\partial t}+\frac{\partial s(t, a)}{\partial a} \geq-\mu s(t, a)-\beta(a) f_{s}^{\prime}\left(\Lambda \pi(a), \frac{\Lambda}{\mu}\right) s(t, a) .
$$

Integrating this equality with the boundary equation $s(t, 0)=\Lambda$ yields

$$
s(t, a) \geq \Lambda e^{-\mu a} e^{-\int_{0}^{a} \beta(s) f_{s}^{\prime}\left(\Lambda \pi(s), \frac{\Lambda}{\mu}\right) d s}>0 .
$$

Second, we claim that $I(t)$ is identically zero if there exists some $t_{0}$ such that $I\left(t_{0}\right)=0$. From the third equation of (5), we follow that

$$
I^{\prime}(t) \leq \int_{0}^{\infty} \beta(a) f_{I}^{\prime}(s(t, a), 0) d a I(t)-(\mu+\delta) I(t)
$$

Solving the above inequality, we have

$$
I(t) \leq I\left(t_{0}\right) e^{\int_{t_{0}}^{t} \int_{0}^{\infty} \beta(a) f_{I}^{\prime}(s(\xi, a), 0) d a d \xi} e^{-(\mu+\delta)\left(t-t_{0}\right)}=0
$$

By the nonnegativity of $I(t)$, we conclude that $I(t)$ is identically zero for all $t \geq t_{0}$. Similarly, we repeat this process and conclude that $I(t)=0$ for all $t<t_{0}$. Consequently, our claim is true.

Finally, we show that $I(t)$ is strictly positive if there does not exist some $t_{0} \in \mathbb{R}$ such that $I(t 0)=0$. By way of contradiction, we redo the second process and arrive at the strict positivity of $I(t)$ for all $t \in \mathbb{R}$.

By Propositions 4.2, 2.5, and 4.3, together with Theorem 3.2 in [25], the following theorem holds.

Theorem 4.4. System (5) is uniformly strongly $\rho$-persistent if $\mathscr{R}_{0}>1$. 
Corollary 4.1. Suppose $\mathscr{R}_{0}>1$ and $I_{0}>0$. Let $(s(t, a), I(t))$ be a total trajectory in $\mathscr{A}$. Then, there exists an $\varepsilon_{0}>0$ such that $s(t, a)>\varepsilon_{0} \pi(a)$ and $I(t)>\varepsilon_{0}$ for all $t \in \mathbb{R}$.

Proof 8. First of all, we claim that $I(t)>0$ for all $t \in \mathbb{R}$. Otherwise, suppose that there exists

$$
t_{0}=\sup _{t \in[-\infty, 0)}\{t \in \mathbb{R} \mid I(t)=0\}
$$

By the assumption of $I_{0}>0$, we conclude that for all $t \in$ $\left[0, t_{0}\right), I(t)>0$, and $I\left(t_{0}\right)=0$. Then by the virtue of the function $f$ with respect to $I$, we have

$$
I^{\prime}\left(t_{0}\right)=\int_{0}^{\infty} \beta(a) f\left(s\left(t_{0}, a\right), I\left(t_{0}\right)\right) d a-(\mu+\delta) I\left(t_{0}\right)=0,
$$

which is a contradiction with $I^{\prime}\left(t_{0}\right)>0$. We repeat this process and obtain $I(t)>0$ for all $t \in \mathbb{R}$. In review of Theorem 4.4 , we immediately have that there exists some positive constant $\epsilon_{1}>0$ such that $I(t)>\epsilon_{1}$. On the other hand, for such $\epsilon_{1}>0$, we recall the process of 4.3 to obtain $s(t, a) \geq \epsilon_{2} \pi(a)$ for $t \in \mathbb{R}$ and $a \in R_{+}$. Hence, $\epsilon_{0}=\min \left\{\epsilon_{1}, \epsilon_{2}\right\}$ is the value that we are looking for and

$$
s(t, a) \geq \epsilon_{0} \pi(a), I(t) \geq \epsilon_{0} .
$$

From Theorem 5.7 in [21], we have the following result.

Theorem 4.5. Suppose Assumptions 1.1 and 1.2 hold. If the basic reproduction number $\mathscr{R}_{0}>1$, then there exists a compact attractor $\mathscr{A}$ that attracts all solutions with initial condition belonging to $\Gamma \backslash \Gamma_{0}$.

For constructing the Lyapunov functionals to get the global stability of the steady states, we define $\varphi:(0, \infty) \rightarrow \mathbb{R}$ as

$$
\varphi(x)=x-1-\ln x
$$

It is well known that $\varphi$ attains a global minimum only at 1 with $\varphi(1)=0$ and $\varphi(x)>0$ for $x \neq 1$. In order to guarantee a well definition of $\varphi$, we give the following proposition.

Proposition 4.6. For all $\left(s_{0}(\cdot), I_{0}\right)^{\mathrm{T}} \in \mathscr{A}$, the following estimation holds.

$$
\frac{s(t, a)}{s^{*}(a)} \geq \frac{\epsilon_{0}}{\Lambda}, \frac{I(t)}{I^{*}(t)} \geq \frac{\epsilon_{0}}{I^{*}},
$$

for all $t \in \mathbb{R}$ and $a \in \mathbb{R}_{+}$.
Proof 9. This is a direct result from Corollary 4.1. We omit the details.

Theorem 4.7. Suppose $\mathscr{R}_{0}>1$ and (A3) of Assumption 1.1 hold, then the disease-free steady state $E_{0}=\left(s^{0}(a), 0\right)=(\Lambda \pi$ $(a), 0)$ is globally asymptotically stable.

Proof 10. Define a Lyapunov function as

$$
V(t)=V_{s}(t)+V_{I}(t)
$$

where $V_{s}(t)=\int_{0}^{\infty} s^{0}(a) \varphi\left(s(t, a) / s^{0}(a)\right) d a$, and $V_{I}=I(t)$. Deviating $V_{s}(t)$ along the solution of system (5) yields

$$
\begin{aligned}
\frac{d V_{S}(t)}{d t} & =\int_{0}^{\infty} s^{0}(a)\left(\frac{1}{s^{0}(a)}-\frac{1}{s(t, a)}\right) \frac{\partial s(t, s)}{\partial t} d a \\
= & \int_{0}^{\infty} s^{0}(a)\left(\frac{1}{s^{0}(a)}-\frac{1}{s(t, a)}\right) \\
& \cdot\left[-\frac{\partial s(t, a)}{\partial a}-\mu s(t, a)-\beta(a) f(s(t, a), I(t))\right] d a .
\end{aligned}
$$

On the other hand,

$$
\begin{aligned}
\frac{\partial \varphi\left(s(t, a) / s^{0}(a)\right)}{\partial a}= & \frac{\partial}{\partial a}\left(\frac{s(t, a)}{s^{0}(a)}-1-\ln \frac{s(t, a)}{s^{0}(a)}\right) \\
= & \frac{\partial}{\partial a}\left(\frac{s(t, a)}{s^{0}(a)}\right)-\frac{\partial}{\partial a} \ln \frac{s(t, a)}{s^{0}(a)} \\
= & \frac{s_{a}^{\prime}(t, a) s^{0}(a)-s(t, a) s_{a}^{0}(a)}{\left(s^{0}(a)\right)^{2}} \\
& -\frac{s^{0}(a)}{s(t, a)} \frac{s_{a}^{\prime}(t, a) s^{0}(a)-s(t, a) s_{a}^{0 \prime}(a)}{\left(s^{0}(a)\right)^{2}} \\
= & \left(\frac{1}{s^{0}(a)}-\frac{1}{s(t, a)}\right) s_{a}^{\prime}(t, a)-s(t, a) \frac{s_{a}^{0 \prime}(a)}{s^{0}(a)} \\
& \cdot\left(\frac{1}{s^{0}(a)}-\frac{1}{s(t, a)}\right) s_{a}^{\prime}(t, a) \\
& +\left(\frac{1}{s^{0}(a)}-\frac{1}{s(t, a)}\right) \mu s(t, a) .
\end{aligned}
$$

We substitute (82) into (81) and obtain

$$
\begin{aligned}
\frac{d V_{S}(t)}{d t} & =\int_{0}^{\infty} s^{0}(a)\left[-\frac{\partial \varphi\left(s(t, a) / s^{0}(a)\right)}{\partial a}-\left(\frac{1}{s^{0}(a)}-\frac{1}{s(t, a)}\right) \beta(a) f(s(t, a), I(t))\right] d a \\
& =-\left.s^{0}(a) \varphi\left(\frac{s(t, a)}{s^{0}(a)}\right)\right|_{0} ^{\infty}-\mu \int_{0}^{\infty} s^{0}(a) g\left(\frac{s(t, a)}{s^{0}(a)}\right) d a-\int_{0}^{\infty} \beta(a)\left(1-\frac{s^{0}(a)}{s(t, a)}\right) f(s(t, a), I(t)) d a .
\end{aligned}
$$


Deviating $V_{I}(t)$ with respect to time $t$ yields

$$
\frac{d V_{I}(t)}{d t}=\int_{0}^{\infty} \beta(a) f(s(t, a), I(t)) d a-(\mu+\delta) I(t) .
$$

Substituting them into the deviation of $V$ gives

$$
\begin{aligned}
\frac{d V(t)}{d t}= & \frac{d V_{s}(t)}{d t}+\frac{d V_{I}(t)}{d t} \\
= & -\mu \int_{0}^{\infty} s^{0}(a) \varphi\left(\frac{s(t, a)}{s^{0}(a)}\right) d a \\
& +\int_{0}^{\infty} \beta(a) \frac{s^{0}(a)}{s(t, a)} f(s(t, a), I(t)) d a-(\mu+\delta) I(t) \\
\leq & -\mu \int_{0}^{\infty} s^{0}(a) \varphi\left(\frac{s(t, a)}{s^{0}(a)}\right) d a \\
& +\left[\int_{0}^{\infty} \beta(a) \frac{\partial f\left(s^{0}(a), 0\right)}{\partial I} d a-(\mu+\delta)\right] I(t) \\
= & -\mu \int_{0}^{\infty} s^{0}(a) \varphi\left(\frac{s(t, a)}{s^{0}(a)}\right) d a+(\mu+\delta)\left(\mathscr{R}_{0}-1\right) I(t),
\end{aligned}
$$

where we use $\left(s^{0}(a)\right) /(s(t, a)) \leq\left(f\left(s^{0}(a), I(t)\right)\right) /(f(s(t, a), I$ $(t))$ ) by (A3) of Assumption 1.1. Therefore, if $\mathscr{R}_{0}>1$, then $V^{\prime}(t) \leq 0$. Since $V(t)$ is bounded on $g(\cdot)$, the alpha limit set of $g(\cdot)$ must be contained in $\mathscr{M}$, the largest invariant subset of $\{(d V(t)) / d t=0\}=\left\{E_{0}\right\}$.

In order to establish the global stability of the endemic steady state, we need the additional assumption for nonlinear function $f$.

Assumption 4.1. For $s(t, a)>0$,

$$
\begin{aligned}
\frac{x}{I^{*}} & <\frac{s^{*}(a) f(s(t, a), x)}{s(t, a) f\left(s^{*}(a), I^{*}\right)}<1, \quad 0<x<I^{*}, \\
1 & <\frac{s^{*}(a) f(s(t, a), x)}{s(t, a) f\left(s^{*}(a), I^{*}\right)}<\frac{x}{I^{*}}, \quad x \geq I^{*} .
\end{aligned}
$$

Now, we are in the position to prove the following result.

Theorem 4.8. Suppose that $\mathscr{R}_{0}>1$ and Assumption 4.1 hold, then the endemic steady state $E^{*}$ is globally asymptotically stable in $\Gamma_{0}$.

Proof 11. By Theorem 3.2 and Theorem 4.5, it suffices to show $\mathscr{A}=\left\{E^{*}\right\}$. Let $g(t)=(s(t, \cdot), I(t))$ be a total trajectory in $\mathscr{A}$. By Corollary 4.1, there exists $\epsilon_{0}>0$, for any $t \in \mathbb{R}$ and $a \in \mathbb{R}_{+}$, such that $0 \leq \varphi(x)<\epsilon_{0}$ for $x=s(t, a) / s^{*}(a)$ and $I(t) /$ $I^{*}$. Define

$$
V(t)=V_{s}(t)+V_{I}(t),
$$

where $V_{s}(t)=\int_{0}^{\infty} s^{*}(a) \varphi\left((s(t, a)) /\left(s^{*}(a)\right)\right) d a$ and $V_{I}(t)=I^{*}$ $\varphi\left((I(t)) / I^{*}\right)$. Then $V(t)$ is bounded and well defined based on Proposition 4.6.
Next, we show that the upper right derivative $(d V(t)) / d t$ along the solution is nonpositive. We firstly have

$$
\begin{aligned}
\frac{d V_{s}(t)}{d t}= & \int_{0}^{\infty} s^{*}(a)\left(\frac{1}{s^{*}(a)}-\frac{1}{s(t, a)}\right) \frac{\partial s(t, a)}{\partial t} d a \\
= & -\int_{0}^{\infty} s^{*}(a)\left(\frac{1}{s^{*}(a)}-\frac{1}{s(t, a)}\right) \frac{\partial s(t, a)}{\partial t} \\
& -\int_{0}^{\infty} s^{*}(a)\left(\frac{1}{s^{*}(a)}-\frac{1}{s(t, a)}\right) \\
& \cdot[\mu s(t, a)+\beta(a) f(s(t, a), I(t))] d a .
\end{aligned}
$$

Furthermore, it follows from

$$
\frac{\partial}{\partial a} g\left(\frac{s_{j}(t, a)}{s^{*}(a)}\right)=\left(\frac{1}{s^{*}(a)}-\frac{1}{s(t, a)}\right)\left\{\frac{\partial}{\partial a} s(t, a)-\frac{s(t, a)}{s^{*}(a)} \frac{d}{d a} s^{*}(a)\right\} .
$$

Then,

$$
\begin{aligned}
\frac{d V_{s}(t)}{d t}= & -\int_{0}^{\infty} s^{*}(a) \frac{\partial}{\partial a} g\left(\frac{s(t, a)}{s^{*}(a)}\right) d a \\
& +\int_{0}^{\infty} \beta(a) f\left(s^{*}(a), I^{*}\right)\left(1-\frac{s^{*}(a)}{s(t, a)}\right) \\
& \cdot\left[\frac{s(t, a)}{s^{*}(a)}-\frac{f(s(t, a), I(t))}{f\left(s^{*}(a), I^{*}\right)}\right] d a .
\end{aligned}
$$

Differentiating $V_{I}$ with respect to $t$, we obtain $\frac{d V_{I}(t)}{d t}=\int_{0}^{\infty} \beta(a) f\left(s^{*}(a), I^{*}\right)\left(1-\frac{I^{*}}{I(t)}\right)\left[\frac{f(s(t, a), I(t))}{f\left(s^{*}(a), I^{*}\right)}-\frac{I(t)}{I^{*}}\right] d a$.

Summing up $\left(d V_{s}(t)\right) / d t$ and $\left(d V_{I}(t)\right) / d t$ together with the expressions of $s(t, a)$ and $s^{*}(a)$ arrives at

$$
\begin{aligned}
\frac{d V(t)}{d t}= & -\left.s^{*}(a) \varphi\left(\frac{s(t, a)}{s(t)}\right)\right|_{0} ^{\infty}+\int_{0}^{\infty} \frac{d s^{*}(a)}{d a} \varphi\left(\frac{s(t, a)}{s(t)}\right) d a \\
& +\int_{0}^{\infty} \beta(a) f\left(s^{*}(a), I^{*}\right) \times\left[\frac{s^{*}(a)}{s(t, a)}+\frac{s^{*}(a) f(s(t, a), I(t))}{s(t, a) f\left(s^{*}(a), I^{*}\right)}\right. \\
= & \left.-\frac{I(t)}{I^{*}}-\frac{I^{*}}{I(t)} \frac{f(s(t, a), I(t))}{f\left(s^{*}(a), I^{*}\right)}\right] d a \\
& +\int_{0}^{\infty} s^{*}(a) \varphi\left(\frac{s(t, a)}{s^{*}(a)}\right) d a-\int_{0}^{\infty} \beta(a) f\left(s^{*}(a), I^{*}\right) \varphi\left(\frac{s(t, a)}{s^{*}(a)}\right) d a \\
= & -\mu \int_{0}^{\infty} s^{*}(a) \varphi\left(\frac{s(t, a)}{s^{*}(a)}\right) d a-\int_{0}^{\infty} \beta\left(\frac{s^{*}(a)}{s(t, a)}\right)+\varphi\left(\frac{s^{*}(a) f(s(t, a), I(t)}{s(t, a) f\left(s^{*}(a), I^{*}\right)}\right) \\
& \left.\cdot\left(\frac{I^{*}}{I(t)} \frac{f(s(t, a), I(t))}{f\left(s^{*}(a), I^{*}\right)}\right) d a+\int_{0}^{\infty} \beta\left(\frac{I(t)}{I^{*}}\right)-\varphi\left(\frac{I^{*}}{I(t)} \frac{f(s(t, a), I(t))}{f\left(s^{*}(a), I^{*}\right)}\right)\right] d a \\
& \cdot\left[\varphi\left(\frac{s^{*}(a) f(s(t, a), I)}{s(t, a) f\left(s^{*}(a), I^{*}\right)}\right)-\varphi\left(\frac{I(t)}{I^{*}}\right)\right] d a .
\end{aligned}
$$




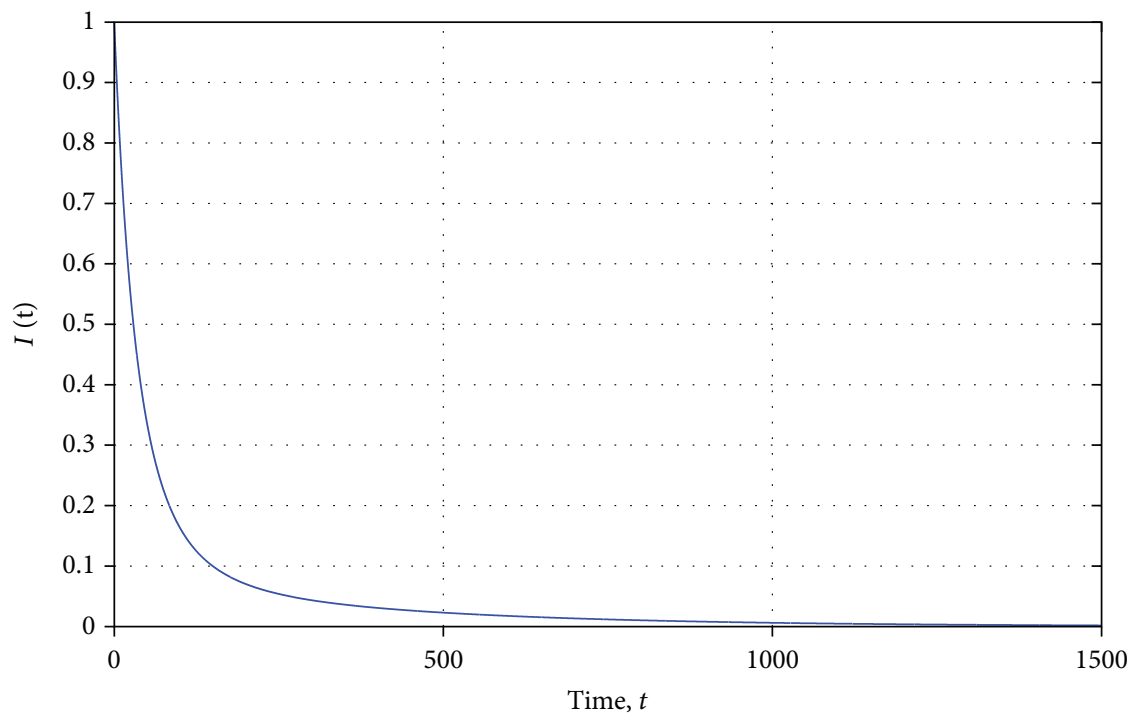

$\beta=0.0035$

(a)

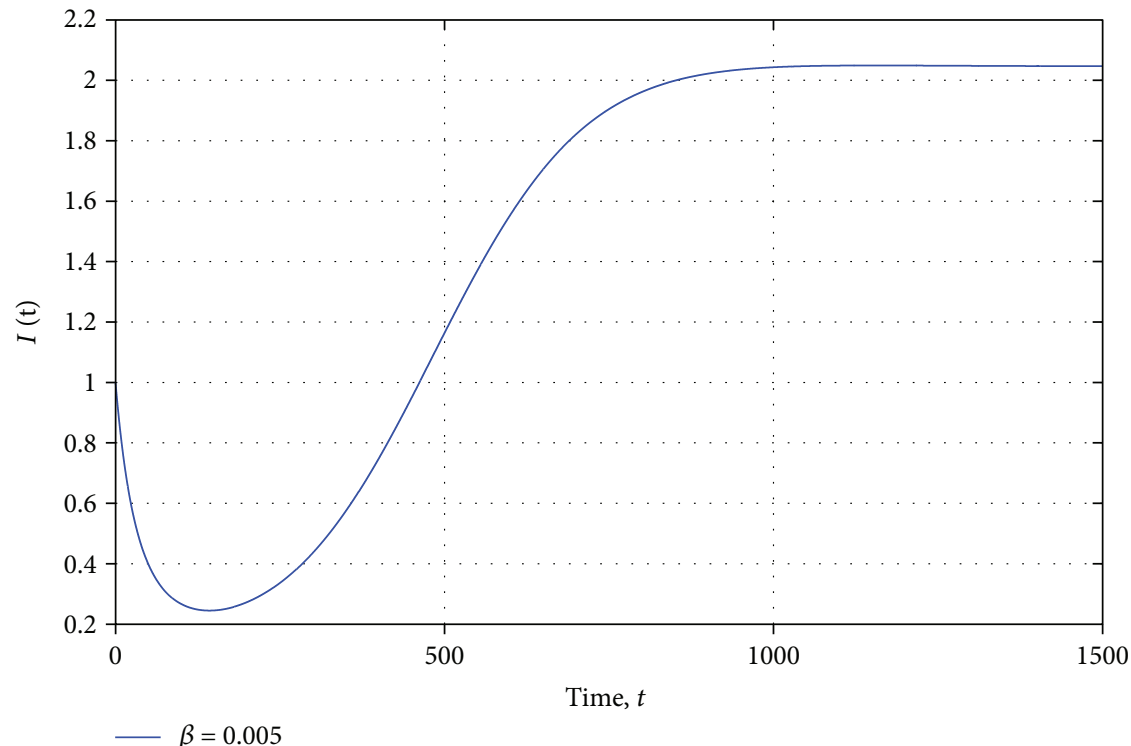

(b)

FIGURE 1: The evolution of the density of the infected $I(t)$ with parameters listed in text. (a) $\beta=0.00035$ and $\mathscr{R}_{0} \simeq 0.9149<1$. (b) $\beta=0.0005$ and $\mathscr{R}_{0} \approx 1.3016$.

In the following, we show that

$$
H(t, a)=\varphi\left(\frac{s^{*}(a) f(s(t, a), I(t))}{s(t, a) f\left(s^{*}(a), I^{*}\right)}\right)-\varphi\left(\frac{I(t))}{I^{*}}\right) .
$$

This, together with Assumption 4.1, implies that $H(t, a)<0$ and then $(d V(t)) / d t \leq 0$. So that $V(t)$ is nonincreasing. Since $V(t)$ is bounded on $g(\cdot)$, the $\omega$-limit set of $g(\cdot)$ must be contained in $\mathscr{M}$, the largest invariant subset of $\{(d V(t)) /$ $d t=0\}$. It follows from $(d V(t)) / d t=0$ that $s(t, a)=s^{*}(a)$ and $\quad\left(s^{*}(a) f(s(t, a), I(t))\right) /\left(s(t, a) f\left(s^{*}(a), I^{*}\right)\right)=(I(t)) / I^{*}$. Immediately, we have

$$
\frac{f(s(t, a), I(t))}{f\left(s^{*}(a), I^{*}\right)}=\frac{I(t)}{I^{*}},
$$

for $t \in \mathbb{R}$ and $a \in \mathbb{R}_{+}$. This, together with the first equation of system (5), yields $f\left(s^{*}(a), I(t)\right)=f\left(s^{*}(a), I^{*}\right)$ for all $t \in \mathbb{R}$ and $a \in \mathbb{R}_{+}$. By the virtue of $f(x, y)$ stated in Assumption (A1), we have that $I(t)=I^{*}$ for all $t$. Therefore, $\mathscr{M}=\left\{E^{*}\right\}$.

The above analysis indicates that the $\omega$-limit set of $g(\cdot)$ consists of just the endemic steady state $E^{*}$ and hence $V$ $(g(t)) \geq V\left(E^{*}\right)$ for all $t \in \mathbb{R}$. Thus, $\mathscr{A}=\left\{E^{*}\right\}$. 


\section{Numerical Simulation}

In this section, we carry out numerical simulations to verify our theoretical results. To show the threshold property for system (1), we pick up the parameters as follows:

$$
\Lambda=0.8, \mu=0.01, \gamma=0.01, \delta=0.01,
$$

and

$$
\beta(a)= \begin{cases}\beta, & t \geq \tau, \\ 0, & t<\tau,\end{cases}
$$

where $\beta$ is varied as our demands. The incidence rate takes in the form of

$$
f(x, y)=\frac{x}{1+\alpha y},
$$

where $\alpha$ is the maturate rate and we fix $\alpha=0.5$. The initial condition is chosen as

$$
s_{0}(a)=\Lambda e^{-\mu a}, I_{0}=1 .
$$

First, we pick up the transmission rate value as $\beta=0.0035$. Then, we directly estimate the basic reproduction number $\mathscr{R}_{0}=0.9149<1$. Theorem 4.7 indicates the global stability of disease-free equilibrium $E_{0}$. Figure 1(a) shows that the disease-free steady state $E_{0}$ decreasingly converges to zero. Second, we take $\beta=0.005$ and calculate $\mathscr{R}_{0}=1.3016>1$. Theorem 4.8 and Figure $1(\mathrm{~b})$ show that the endemic steady state $E^{*}\left(s^{*}(a), I^{*}\right)$ is asymptotically stable.

\section{Discussion}

In this paper, we proposed an SIR model with age structure in susceptibility and a general incidence rate. We gave the existence, uniqueness, and regularity of the solution of system (5). We calculated the basic reproduction number, which is the spectral radius of the next-generation operator. Based on the assumptions on the incidence function and the parameters, we obtain the asymptotical smoothness of the system and existence, uniqueness, local stability, and global stability of the steady states. The interesting thing is that the global behavior of system (5) is rigorously proved by constructing Lyapunov functional method.

Incidence rate plays an important role in investigating the disease transmission pattern. As mentioned in Introduction, bilinear type and nonlinear type had been studied in many literatures. We used a general function $f(s, I)$ to extend the two classical types and so it can cover some existing results $[8,9]$. Some complex dynamics (Hopf bifurcation [26], backward bifurcation [27], homoclinics [28], and so on) inducing by complex incidence rate had been investigated. While in this paper, we just focus on the global dynamics based on some rigorous limitations for the nonlinear incidence rate $f(s, I)$. Oscillation is a nature phenomenon in realistic life, and exploring the intrinsic mechanism has been become a hot topic in epidemic modelling processes. In the future, we will turn our focus on this aspect to discover some substantial mechanisms to result in disease oscillations.

\section{Data Availability}

Our data is artificial selection to support our theoretical results.

\section{Conflicts of Interest}

The authors declare that there is no conflict of interest regarding the publication of this paper.

\section{Acknowledgments}

Research is partially supported by the National Natural Science Foundation of China (no. 61573016 and no. 61203228), the Shanxi Scholarship Council of China (2015-094), the Shanxi Scientific Data Sharing Platform for Animal Diseases, and the Startup foundation for High-level Personal of Shanxi University.

\section{References}

[1] J. Wang, M. Guo, and S. Liu, "SVIR epidemic model with age structure in susceptibility, vaccination effects and relapse," IMA Journal of Applied Mathematics, vol. 82, no. 5, pp. 945970, 2017.

[2] J. Wang, X. Liu, T. Kuniya, and J. Pang, "Global stability for multi-group SIR and SEIR epidemic models with agedependent susceptibility," Discrete \& Continuous Dynamical Systems - B, vol. 22, no. 7, pp. 2795-2812, 2017.

[3] W. O. Kermack and A. G. McKendrick, "Contributions to the mathematical theory of epidemics-I," Bulletin of Mathematical Biology, vol. 53, no. 1-2, pp. 33-55, 1991.

[4] W. O. Kermack and A. G. McKendrick, "Contributions to the mathematical theory of epidemics-II. The problem of endemicity," Bulletin of Mathematical Biology, vol. 53, no. 1-2, pp. 57-87, 1991.

[5] V. Capasso and G. Serio, "A generalization of the KermackMcKendrick deterministic epidemic model," Mathematical Biosciences, vol. 42, no. 1-2, pp. 43-61, 1978.

[6] G. Huang, Y. Takeuchi, W. Ma, and D. Wei, "Global stability for delay SIR and SEIR epidemic models with nonlinear incidence rate," Bulletin of Mathematical Biology, vol. 72, no. 5, pp. 1192-1207, 2010.

[7] A. Korobeinikov and P. K. Maini, "Non-linear incidence and stability of infectious disease models," Mathematical Medicine and Biology, vol. 22, no. 2, pp. 113-128, 2005.

[8] A. Korobeinikov, "Lyapunov functions and global stability for SIR and SIRS epidemiological models with non-linear transmission," Bulletin of Mathematical Biology, vol. 68, no. 3, pp. 615-626, 2006.

[9] P. Magal, C. C. McCluskey, and G. F. Webb, "Lyapunov functional and global asymptotic stability for an infection-age model," Applicable Analysis, vol. 89, no. 7, pp. 1109-1140, 2010.

[10] X. Duan, S. Yuan, Z. Qiu, and J. Ma, "Global stability of an SVEIR epidemic model with ages of vaccination and latency," Computers \& Mathematcs with Applications, vol. 68, no. 3, pp. 288-308, 2014. 
[11] H. Gulbudak and M. Martcheva, "A structured avian influenza model with imperfect vaccination and vaccine induced asymptomatic infection," Bulletin of Mathematical Biology, vol. 76, no. 10, pp. 2389-2425, 2014.

[12] Y. Chen, J. Yang, and F. Zhang, "The global stability of an SIRS model with infection age," Mathematical Biosciences \& Engineering, vol. 11, no. 3, pp. 449-469, 2014.

[13] C. C. McCluskey, "Global stability for an SIR epidemic model with delay and nonlinear incidence," Nonlinear Analysis: Real World Applications, vol. 11, no. 4, pp. 3106-3109, 2010.

[14] Y. Chen, S. Zou, and J. Yang, "Global analysis of an SIR epidemic model with infection age and saturated incidence," Nonlinear Analysis: Real World Applications, vol. 30, pp. 16-31, 2016.

[15] B. Soufiane and T. M. Touaoula, "Global analysis of an infection age model with a class of nonlinear incidence rates," Journal of Mathematical Analysis and Applications, vol. 434, no. 2, pp. 1211-1239, 2016.

[16] J. Yang, X. Li, and F. Zhang, "Global dynamics of a heroin epidemic model with age structure and nonlinear incidence," International Journal of Biomathematics, vol. 9, no. 3, 2016.

[17] J. L. Wang, R. Zhang, and T. Kuniya, "Global dynamics for a class of age-infection HIV models with nonlinear infection rate," Journal of Mathematical Analysis and Applications, vol. 432, no. 1, pp. 289-313, 2015.

[18] H. R. Thieme, "Semiflows generated by Lipschitz perturbations of non-densely defined operators," Differential Integral Equations, vol. 3, no. 6, pp. 1035-1066, 1990.

[19] P. Magal, "Compact attractors for time-periodic agestructured population models," Electronic Journal of Differential Equations, vol. 2001, no. 65, pp. 1-35, 2001.

[20] J. Hale, Asymptotic Behavior of Dissipative Systems, AMS, Providence, RI, USA, 1988.

[21] H. L. Smith and H. R. Thieme, "Dynamical systems and population persistence," Graduate Studies in Mathematics, vol. 118, 2011.

[22] O. Diekmann, H. Heesterbeek, and T. Britton, Mathematical Tools for Understanding Infectious Disease Dynamics, Princeton University Press, Princeton, NJ, USA, 2013.

[23] H. Inaba, "The Malthusian parameter and $R_{0}$ for heterogeneous populations in periodic environments," Mathematical Biosciences \& Engineering, vol. 9, no. 2, pp. 313-346, 2012.

[24] J. Yang and F. Xu, "Global stability of two SIS epidemic meanfield models on complex networks: Lyapunov functional approach," Journal of the Franklin Institute, vol. 355, pp. 6763-6779, 2018.

[25] H. R. Thieme, "Uniform persistence and permanence for nonautonomous semiflows in population biology," Mathematical Biosciences, vol. 166, no. 2, pp. 173-201, 2000.

[26] Z. H. Liu and R. Yuan, "Zero-Hopf bifurcation for an infection-age structured epidemic model with a nonlinear incidence rate," Science China Mathematics, vol. 60, no. 8, pp. 1371-1398, 2017.

[27] M. A. Khan, Y. Khan, and S. Islam, "Complex dynamics of an SEIR epidemic model with saturated incidence rate and treatment," Physica A: Statistical Mechanics and its Applications, vol. 493, pp. 210-227, 2018.

[28] F. Berezovsky, G. Karev, B. Song, and C. Castillo-Chavez, "A simple epidemic model with surprising dynamics," Mathematical Biosciences \& Engineering, vol. 2, no. 1, pp. 133-152, 2005. 


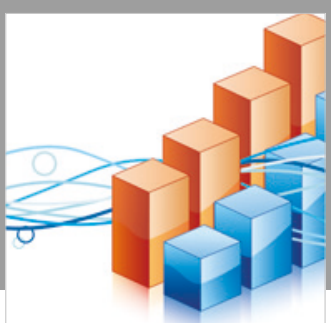

Advances in

Operations Research

\section{-n-m}
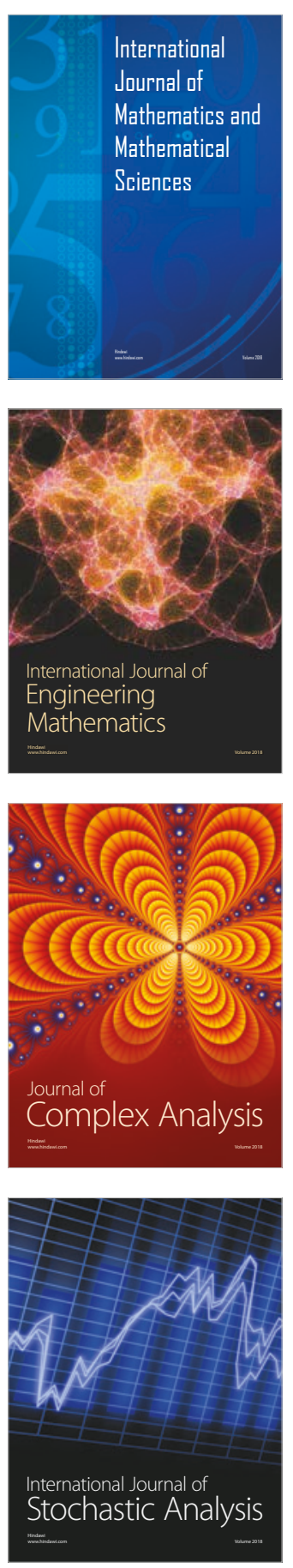
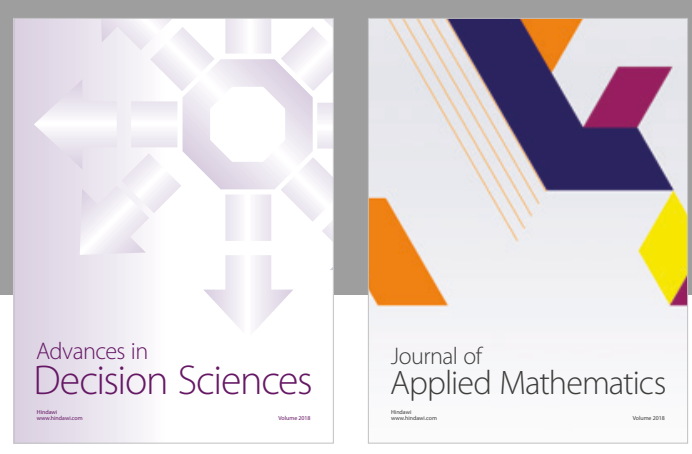

Journal of

Applied Mathematics
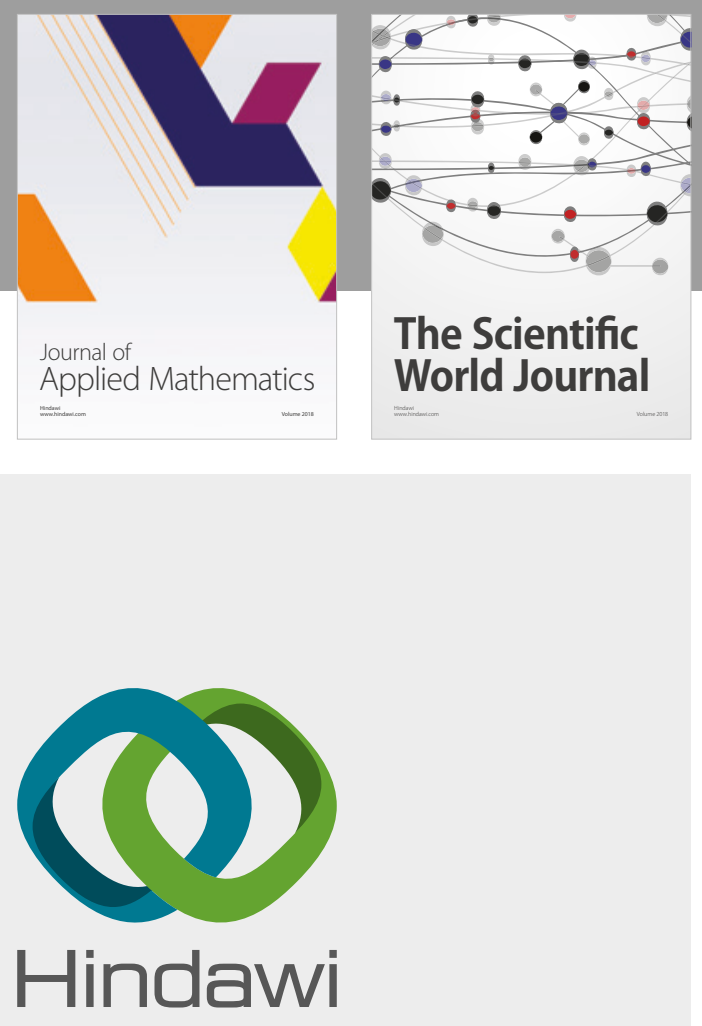

Submit your manuscripts at

www.hindawi.com

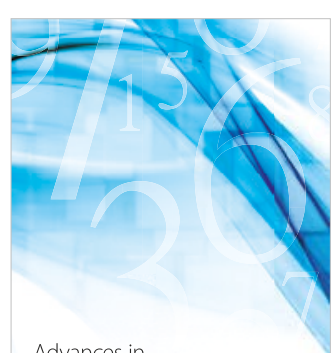

Advances in
Numerical Analysis
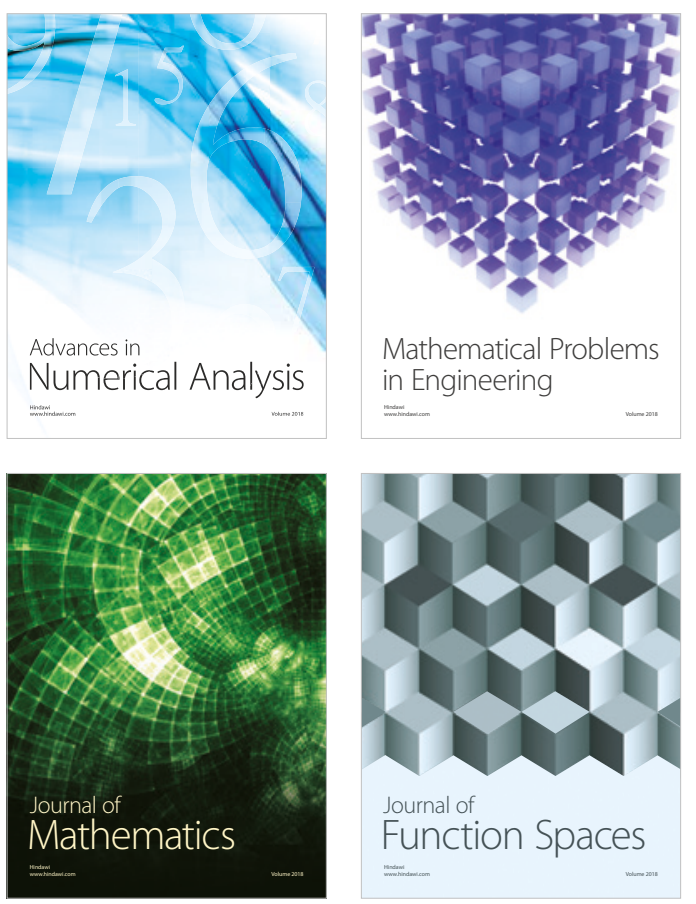

Mathematical Problems in Engineering

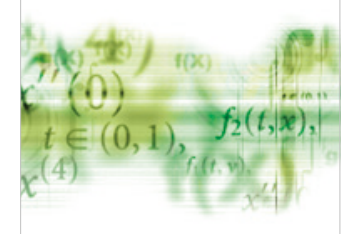

International Journal of

Differential Equations

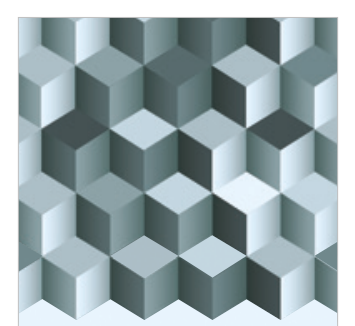

Journal of

Function Spaces
The Scientific

World Journal

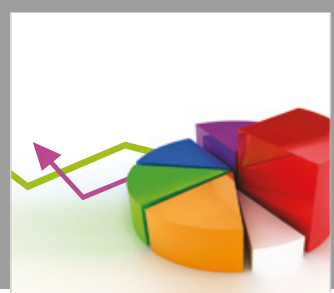

Journal of

Probability and Statistics
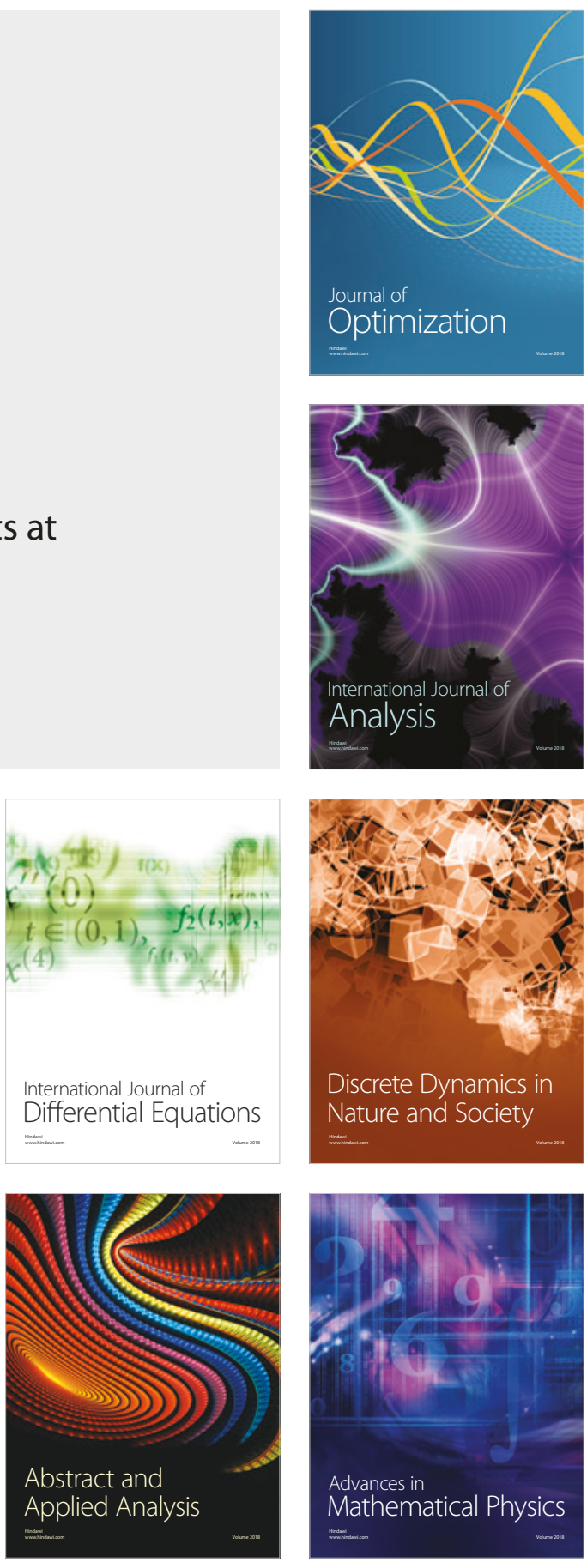